\title{
Activity-Dependent Remodeling of Drosophila Olfactory Sensory Neuron Brain Innervation during an Early-Life Critical Period
}

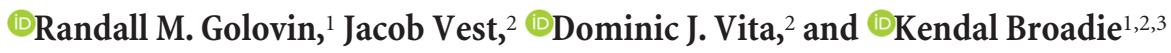 \\ ${ }^{1}$ Vanderbilt Brain Institute, ${ }^{2}$ Department of Biological Sciences, and ${ }^{3}$ Department of Cell and Developmental Biology, Vanderbilt University and Medical \\ Center, Nashville, Tennessee 37235
}

Critical periods are windows of development when the environment has a pronounced effect on brain circuitry. Models of neurodevelopmental disorders, including autism spectrum disorders, intellectual disabilities, and schizophrenia, are linked to disruption of critical period remodeling. Critical periods open with the onset of sensory experience; however, it remains unclear exactly how sensory input modifies brain circuits. Here, we examine olfactory sensory neuron (OSN) innervation of the Drosophila antennal lobe of both sexes as a genetic model of this question. We find that olfactory sensory experience during an early-use critical period drives loss of OSN innervation of antennal lobe glomeruli and subsequent axon retraction in a dose-dependent mechanism. This remodeling does not result from olfactory receptor loss or OSN degeneration, but rather from rapid synapse elimination and axon pruning in the target olfactory glomerulus. Removal of the odorant stimulus only during the critical period leads to OSN reinnervation, demonstrating that remodeling is transiently reversible. We find that this synaptic refinement requires the OSN-specific olfactory receptor and downstream activity. Conversely, blocking OSN synaptic output elevates glomeruli remodeling. We find that GABAergic neurotransmission has no detectable role, but that glutamatergic signaling via NMDA receptors is required for OSN synaptic refinement. Together, these results demonstrate that OSN inputs into the brain manifest robust, experience-dependent remodeling during an early-life critical period, which requires olfactory reception, OSN activity, and NMDA receptor signaling. This work reveals a pathway linking initial olfactory sensory experience to glutamatergic neurotransmission in the activity-dependent remodeling of brain neural circuitry in an early-use critical period.

Key words: antennal lobe; critical period; development; NMDA receptor; sensory experience; synapse elimination

Significance Statement

Neurodevelopmental disorders manifest symptoms at specific developmental milestones that suggest an intersection between early sensory experience and brain neural circuit remodeling. One classic example is Fragile $\mathrm{X}$ syndrome caused by loss of an RNA-binding translation regulator of activity-dependent synaptic refinement. As a model, Drosophila olfactory circuitry is well characterized, genetically tractable, and rapidly developing, and thus ideally suited to probe underlying mechanisms. Here, we find olfactory sensory neurons are dramatically remodeled by heightened sensory experience during an early-life critical period. We demonstrate removing the olfactory stimulus during the critical period can reverse the connectivity changes. We find that this remodeling requires neural activity and NMDA receptor-mediated glutamatergic transmission. This improved understanding may help us design treatments for neurodevelopmental disorders.

\section{Introduction}

Critical periods are discrete developmental time windows when the brain is especially susceptible to modification by sensory stimuli. Since the classical visual cortex work by Hubel and Wiesel (1970), enormous progress has been made in understanding critical period refinement (LeVay et al., 1980; Hensch, 2005; 
Morishita et al., 2010) and how it goes awry in neurological diseases (Dölen et al., 2007; Contractor et al., 2015; Doll and Broadie, 2015, 2016; Golovin and Broadie, 2016; Doll et al., 2017; Vita and Broadie, 2017). Typically, critical periods open with the onset of sensory experience and close after a defined period of refinement, during which neural circuitry is modified to better respond to the sensory environment (Hensch, 2005). Although initial studies painted a stark black-and-white picture regarding opening and closing of critical periods, it is now known that numerous factors can reopen critical period-like states in mature animals (McGee et al., 2005; Maya Vetencourt et al., 2008; Morishita et al., 2010; Hensch and Bilimoria, 2012; Baroncelli et al., 2016). Critical period work has focused primarily on vertebrate systems, but there are excellent examples of critical periods in invertebrate models (Fielde, 1904; Remy and Hobert, 2005; Doll and Broadie, 2015; Jin et al., 2016). The short generation time and powerful genetic tools available in these systems make them attractive candidates for furthering our understanding of critical periods.

The Drosophila antennal lobe (AL) circuitry is particularly well mapped (Vosshall et al., 2000; Wang et al., 2003; Grabe et al., 2016). In the AL, the axon termini from olfactory sensory neurons (OSNs) synapse onto projection neurons and local interneurons within discrete synaptic glomeruli (Couto et al., 2005; Fishilevich and Vosshall, 2005; Wilson, 2013). OSNs that express the same olfactory receptor innervate the same target AL glomerulus to form synaptic connections with the same projection neurons. Each glomerulus can be individually identified based both on anatomical position and by the expression of the defining specific olfactory receptor (Jefferis et al., 2001, 2002, 2004). The AL circuit is grossly hard-wired with no large-scale changes to the glomerular map, even after complete loss of olfaction (Larsson et al., 2004). Although the overall glomerular map is stable, a growing body of work has shown that individual glomeruli can alter their morphology and functionality in response to the odorant environment (Devaud et al., 2001, 2003a,b; Sachse et al., 2007; S. Das et al., 2011; Acebes et al., 2012; Doll and Broadie, 2015, 2016). Environmental odorants usually bind to multiple sensory odorant receptors with different affinities, which lead to a characteristic AL activity map dependent on different odorant concentrations (Hallem and Carlson, 2006). Thus, olfactory sensory experience can be mapped onto central brain olfactory circuitry.

Seminal early papers demonstrated AL critical period refinement in response to olfactory experience (Devaud et al., 2001, 2003a,b). Later studies showed selective remodeling of the $\mathrm{CO}_{2}$ sensitive glomerulus (Sachse et al., 2007), and local interneuron modulatory roles shaping the critical period (Acebes et al., 2011, 2012). Molecular pathways involved include NMDA-dependent glutamatergic signaling, cAMP signal transduction (dunce, rutabaga, creb), and translational regulation (FMRP, Ataxin2) (Devaud et al., 2001, 2003b; S. Das et al., 2011; Sudhakaran et al., 2014; Doll and Broadie, 2015, 2016). Previous studies have reported that odor activation of glomeruli during the early-life critical period can increase glomerular volume (Sachse et al., 2007; S. Das et al., 2011; Kidd et al., 2015). We tested this result, taking advantage of genetic tools to probe OSN-specific structural and synaptic changes with the commonly used odorant ethyl butyrate (EB) on the strongly activated VM7 glomerulus (DoOR version

Correspondence should be addressed to Kendal Broadie at kendal.broadie@vanderbilt.edu. https://doi.org/10.1523/JNEUROSCI.2223-18.2019

Copyright $\odot 2019$ the authors
2.0) (Münch and Galizia, 2016), but we unexpectedly discovered strongly reduced glomerular volume with critical period exposure. This finding shows that odorants can drive opposing remodeling changes in discrete glomeruli. Synaptic remodeling is reversible, activity-dependent, and requires NMDA receptor signaling. Overall, this study expands our knowledge of mechanisms of critical period circuit remodeling and provides a platform to investigate neuron-specific requirements.

\section{Materials and Methods}

Drosophila genetics. All animals were raised at $25^{\circ} \mathrm{C}$ on standard cornmeal/agar/molasses Drosophila food in a $12 \mathrm{~h}$ light/dark cycling incubator until odorant exposure (see below). The following lines were used in genetic crosses: Or42a-Gal4 (RRID:BDSC_9969) (Fishilevich and Vosshall, 2005); UAS-mCD8::GFP (RRID:BDSC_5137) (Doll and Broadie, 2015); UASmCD8::RFP (RRID:BDSC_32219); Brp-FRT-GFP (Chen et al., 2014); UASFLP1; Or42a-Gal4 (RRID:BDSC_4539/RRID:BDSC_9969); Or42a $a^{\mathrm{FO} 4305}$; Or42a-mCD8::GFP (RRID:BDSC_18758) (Thibault et al., 2004; Stephan et al., 2012); UAS-mCD8::GFP;Or42a-Gal4; UAS-TeTxLC (Wang et al., 2012); UAS-Kir2.1-eGFP (RRID:BDSC_6596) (Baines et al., 2001); UAS-GABAb R3 RNAi (RRID:BDSC_26729) (Flockhart et al., 2006); UAS-NMDAR1 RNAi (RRID:BDSC_41666) (Flockhart et al., 2006); $w g^{l-17}$ (Baker, 1987); UAS-dFz2-DN (Zhang and Carthew, 1998); and UAS-Sgg-DN (RRID:BDSC_5360) (Bourouis, 2002). All genotypes were confirmed with visible markers or PCR. $w^{1118}$ (RRID:BDSC_3605) was used as a genetic background control. Transgenic controls used included $w^{1118}$; UAS-mCD8::GFP/+;Or42a-Gal4/+| and $w^{1118}$; Or42a-mCD8:: GFP/Or42a-mCD8::GFP. Animals of both sexes were used in all studies, except where specifically noted in figure legends. All crosses were transferred to fresh food every 2-3 d, with rearing densities matched between genotypes.

Odorant exposure. Staged animals were sorted as dark pupae into separate vials based on sex, genotype, and odor exposure (except for Fig. $1 C, D$, where animals were aged $7 \mathrm{~d}$ before exposure). A fine wire stainless-steel mesh (Small Parts) was secured with tape over the top of the vial to contain the flies but still allow airflow. Vials were placed in an airtight $3700 \mathrm{ml}$ Glasslock container with either $1 \mathrm{ml} \mathrm{15 \%}$ or $25 \% \mathrm{~EB}$ (Sigma-Aldrich; \% v/v EB in mineral oil) or the vehicle only (mineral oil alone) in a $1.5 \mathrm{ml}$ microcentrifuge tube attached to the side of the chamber. Exposure chambers were placed in temperature-controlled incubators $\left(23^{\circ} \mathrm{C}\right)$ on $12 \mathrm{~h} \mathrm{light/dark}$ cycles. At $18-21 \mathrm{~h}$ after placing vials into the chambers, eclosed flies were rapidly transferred to clean vials with fresh food and placed in clean exposure chambers with freshly made odorants, as above. Except for experiments shown in Figure 3, animals were kept in the odor exposure chambers in incubators for $48 \mathrm{~h}$ and then processed for immunohistochemistry. For Figure 3 experiments, animals were kept in the EB exposure chambers for $24 \mathrm{~h}(1 \mathrm{~d}), 48 \mathrm{~h}(2 \mathrm{~d})$, or $96 \mathrm{~h}$ ( $4 \mathrm{~d}$ ), whereas $3 \mathrm{~d}$ reversals were kept on EB for $24 \mathrm{~h}$ and then transferred to oil for $72 \mathrm{~h}$, and $2 \mathrm{~d}$ reversals were kept on EB for $48 \mathrm{~h}$ and then transferred to oil for $48 \mathrm{~h}$. Testing indicates that exposing animals to odorant from the dark pupae stage causes a more consistent phenotype, indicating that odorant exposure immediately after eclosion is critically important.

Immunohistochemistry imaging. Staged animals were anesthetized on ice for 1-2 min. Brains were then dissected using fine, sharpened forceps (Dumont \#5) in physiological saline ([in mM] as follows: $128 \mathrm{NaCl}, 2$ $\mathrm{KCl}, 4 \mathrm{MgCl}_{2}, 1.8 \mathrm{CaCl}_{2}, 64.6$ sucrose, 5 HEPES, $\mathrm{pH} 7.2$; all reagents from Sigma-Aldrich). After dissection, brains were fixed for $30 \mathrm{~min}$ at room temperature in 4\% PFA (EMS)/4\% sucrose in PBS, pH 7.4 (Invitrogen). Fixed brains were washed $3 \times$ with PBS and then blocked for $1 \mathrm{~h}$ in $1 \%$ BSA (Sigma-Aldrich) in PBS-T $(0.2 \%$ Triton X-100 in PBS; Fisher Chemical). Brains were incubated with primary antibodies diluted in $0.2 \% \mathrm{BSA}$ in PBS-T at $4^{\circ} \mathrm{C}$ overnight $(\sim 14-18 \mathrm{~h})$. The primary antibodies used were as follows: rabbit anti-GFP (Abcam 290; 1:3000 or 1:6000 empirically determined for each aliquot), mouse anti-BRP (Developmental Studies Hybridoma Bank, nc82; 1:50), and rat anti-RFP (Chromotek 5F8; 1:500). The next day, brains were washed $3 \times$ for 20 min with PBS-T and then incubated overnight $(14-18 \mathrm{~h})$ with secondary antibod- 
ies conjugated to fluorescent tags. The secondary antibodies used were as follows: AlexaFluor-488 goat anti-rabbit, AlexaFluor-546 goat antimouse, AlexaFluor-546 goat anti-rat, and AlexaFluor-633 goat antimouse (all used at 1:250). Following secondary incubation, the brains were washed in PBS-T $3 \times$ for $20 \mathrm{~min}$ followed by PBS $1 \times$ for $20 \mathrm{~min}$. Brains were then rinsed with $\mathrm{dH}_{2} \mathrm{O}$ and mounted onto clean glass slides (ProbeOn Microscope Slides, Fisherbrand) with Fluoromount (EMS 17984-25) with a glass coverslip (No. 1.5H, Carl Zeiss). Double-sided adhesive tape (Scotch) was used to raise coverslips above the brains, and clear nail polish (Sally Hansen) was used to seal a coverslip to the slide. For maxillary palps (see Fig. 2), whole proboscises were dissected and processed exactly like the brains, except fixed for $45 \mathrm{~min}$ with longer primary/secondary antibody incubations ( $38-42 \mathrm{~h}$ ). In Figure 4, brains were put through a concentration series of 2,2-thiodiethanol (TDE; Sigma-Aldrich) $10 \%, 25 \%, 50 \%$, and $97 \%$ TDE in PBS for 10 min each, followed by $2 \times$ washes with $97 \%$ TDE in PBS and then mounting in $97 \%$ TDE in PBS as above. Fluorescent images were collected on a 510 META laser-scanning confocal microscope (Carl Zeiss) with either a $40 \times$ or $63 \times$ oil-immersion lens. Images taken with the $40 \times$ objective were collected at $1024 \times 1024$ resolution with a $Z$ slice of $1 \mu \mathrm{m}$ thickness. Images taken with the $63 \times$ objective were collected at $2048 \times 2048$ resolution with a $Z$-slice thickness of $0.8 \mu \mathrm{m}$. The microscope and imaging settings were kept constant within every experiment.

Glomerular measurements. For glomerular volume measurements, an ROI was defined for the maximal borders of the VM7 glomerulus and the FIJI plugin 3D Objects Counter was used to quantify volume (Schindelin et al., 2012) (RRID:SCR_002285). The threshold was chosen to minimize noise. When the signal from the glomerulus was sparse, multiple object volumes were summed to obtain a single data point. Data collected from different days of experiments were normalized to the appropriate control to account for variation between replicates. To count VM7 Bruchpilot (BRP) punctae, the "find maxima" tool in NIH ImageJ (Schneider et al., 2012) was used to identify local maxima above a chosen noise tolerance. The "find maxima stacks" macro in ImageJ allowed quantification for an entire image by using the find maxima function for each slice. BRP intensity measurements were derived from the "histogram function" in ImageJ, which provides pixels at brightness (range: $0-255$ ). To generate intensity values, we used the weighted sum of all pixels, with the number of pixels at each level of brightness multiplied by the brightness value, and the products summed together to generate the overall intensity. Brightness values $<20$ were dropped to account for image background.

Soma measurements. For maxillary palp measurements, blinded $Z$-stack images were analyzed using ImageJ and the Or42a OSN cell bodies manually counted throughout the entire maxillary palp. For the fluorescence intensity measurements, the ROI was defined on a blinded maximum intensity projection of the Or42a OSN cell bodies and proximal labial nerve within the maxillary palp. The ImageJ measurement tool was then used to quantify the mean fluorescence intensity of this ROI.

Western blots. Western blots were performed as reported (Vita and Broadie, 2017), with slight modifications. Staged animals were exposed to oil or EB as described above. After exposure, animals were anesthetized on ice, and maxillary palps were removed in dissecting saline with EDTAfree protease inhibitor (Roche Diagnostics, 04693132001). Palps were placed in $24 \mu$ l RIPA buffer ( $150 \mathrm{~mm}$ sodium chloride, $1 \%$ Triton X-100, $0.5 \%$ sodium deoxycholate, $0.1 \%$ SDS, $50 \mathrm{~mm}$ Tris), immediately snap frozen on dry ice, and stored at $-80^{\circ} \mathrm{C}$ for $<1$ week. Palps in RIPA buffer were then defrosted on ice followed by sonication (Sonifier, Branson, setting $90 \%$ duty, output 2) for 20 s, vortexed (Standard Mini Vortexer, VMR Scientific Products, speed 4) for $5 \mathrm{~s}$, and then centrifuged at 12,000 RPM for $10 \mathrm{~min} ; 12 \mu \mathrm{l}$ of lysate was then transferred to new prechilled tubes, $4 \mu \mathrm{l}$ of NuPage LDS buffer (Invitrogen, NP007) and $0.8 \mu \mathrm{l}$ of 2-mercaptoethanol (Sigma-Aldrich, M7154) were added, and the lysate vortexed as above. Samples were placed at room temperature to incubate for $20 \mathrm{~min}$ and then boiled for $10 \mathrm{~min}$ followed by centrifugation $(14,000$ $\mathrm{RPM}, 10 \mathrm{~min})$. Samples were then loaded into precast NuPage $4-12 \%$ Bis-Tris gels (Invitrogen, NP0336) with NuPage Mes SDS running buffer (Invitrogen, NP002). Buffer in the middle chamber was supplemented with NuPage antioxidant (Invitrogen, NP0005) to ensure 2-mercaptoethanol movement into gel. Samples were run at $150 \mathrm{~V}$ until loading dye exited the gel. Transfers were performed overnight at $4^{\circ} \mathrm{C}$ with constant $30 \mathrm{~mA}$ current. Proteins were transferred to nitrocellulose membranes (PROTRAN, NBA085C001EA) in $4^{\circ} \mathrm{C}$ NuPage transfer buffer (Invitrogen, NP0006-1) supplemented with 20\% methanol (Honeywell, AH2304). Membranes were rinsed with deionized water for $5 \mathrm{~min}$, then air-dried for $1 \mathrm{~h}$. Total protein was analyzed via REVERT total protein stain (LI-COR, 926-11011) according to the manufacturer's instructions. Membranes were then blocked with 2\% powdered milk (instant nonfat dry milk, Kroger) in TBS-T (0.1\% Tween-20, 150 mm sodium chloride, 5 mM potassium chloride, $25 \mathrm{~mm}$ Tris, $\mathrm{pH}$ 7.6) for $1 \mathrm{~h}$ at room temperature with rotation. Primary antibody in $2 \%$ milk in TBS-T was incubated overnight at $4^{\circ} \mathrm{C}$ with rotation. Membranes were then washed $5 \times 6 \mathrm{~min}$ with TBS-T at room temperature with rotation. Secondary antibody in $2 \%$ milk in TBS-T was incubated for $1 \mathrm{~h}$ at room temperature with rotation, then washed as above. Membranes were imaged using an Odyssey imager (LI-COR Biosciences), with intensity measurements taken by Image Studio Lite (LI-COR Biosciences). Bands were normalized to REVRT total protein stain. Primary antibody used was rabbit anti-GFP (Abcam 290, 1:2500). Secondary antibody was goat anti-rabbit 680 (AlexaFluor-680, 1:10,000). Because of differences in transgenic insert composition (1xGFP vs $4 \mathrm{xGFP}$ ), Or42a $>$ mcd8::GFP runs at a smaller molecular weight than Or42a-mcd8::GFP (Couto et al., 2005; Hara et al., 2017).

$q R T-P C R$. As above, staged animals were exposed to EB or vehicle for $48 \mathrm{~h}$ after eclosion, and then proboscises with maxillary palps were dissected free with fine forceps (Dumont) and transferred immediately to 10 $\mu \mathrm{l}$ water with $1 \mathrm{u} / \mu \mathrm{l}$ RNase inhibitor on ice (Applied Biosystems). To enhance signal, animals expressing 2 copies Or42a-Gal4 driving 2 copies of mCD8::GFP were used, with samples from 5 males and 5 females combined from each odorant condition, repeated in three independent replicates. RNA was extracted using the RNeasy Micro Kit (QIAGEN) following the manufacturer's instructions. cDNA synthesis from total RNA and qRT-PCR was performed using the Power SYBR Green RNAto- $\mathrm{C}_{\mathrm{T}}$ 1-Step Kit (Applied Biosystems) following the manufacturer's instructions, except that RNase inhibitor was added to the PCR reaction at $1 \mathrm{u} / \mu \mathrm{l}$. For each reaction, $1 \mathrm{ng}$ of total RNA was used with the following primers: 5'-AAGAAAAACCGAAGTGCGCC-3' and 5'-AGTCAGCGG AGACCTTTTGG-3' for Gal4 (DNA-binding domain), 5'-TTTTGCG ATTTGTTGACTGC CT- ${ }^{\prime}$ ' and 5' $^{\prime}$-TTAGGGTAAAGCCCAGCACC- ${ }^{\prime}$ for Or $42 a, 5^{\prime}$-TCACCCAAATTCTGAGTCCC G-3' ${ }^{\prime}$ and $5^{\prime}$-CATCATGG CGGCAAATCCTG-3' for Or71a, 5' -TACAGGCCCAAGATCGTGAA-3' and $5^{\prime}$-TCTCCTTGCGCTTCTTGGA-3' for $r p 49$ (LaLonde et al., 2006), 5'-CGTTCATGCCACCACCGCTA-3' and 5' -CCACGTCCATCACGCC ACAA-3' for GAPDH2 (Ling and Salvaterra, 2011). Primers were tested and verified to produce a single peak using melting curve analysis. qPCR retractions were run on a Bio-Rad CFX-96 Real-Time System, with PCRs run in duplicate and expression values from each run averaged. Data were analyzed using the $\Delta \Delta$ Ct method (Livak and Schmittgen, 2001). Each expression level was normalized to housekeeping genes GAPDH2 and $r p 49$, with values then normalized to the mean vehicle control.

mini Singlet Oxygen Generator (miniSOG) photoconversion. Photoconversion for electron microscopy was performed as described, with some minor revisions (Strickfaden et al., 2015; Ng et al., 2016). It is imperative that all solutions are made fresh at $\mathrm{pH} 7.4$ for photoconversion. Staged animals expressing membrane-tethered miniSOG (UAS-myr-miniSOG) driven by Or $42 a-G a l 4$ were exposed to oil vehicle or $25 \% \mathrm{~EB}$, and then the brains were dissected as described above. All following steps were performed at room temperature, in a dark chamber, with end-over-end rotation. Immediately following dissection, brains were fixed for $30 \mathrm{~min}$ with $4 \%$ PFA (EMS 15714) in 0.1 m sodium cacodylate (SC, EMS 11652) buffer followed by $2 \times 20$ min washes with $0.1 \mathrm{M} \mathrm{SC}$. Brains were then incubated for $30 \mathrm{~min}$ in a solution of $5 \mathrm{~mm}$ aminotriazole (Sigma-Aldrich A8056) and $50 \mathrm{~mm}$ glycine (Sigma-Aldrich G8898) in $0.1 \mathrm{~m} \mathrm{SC}$ buffer to reduce endogenous peroxidase activity (Strickfaden et al., 2015; Ng et al., 2016), followed by $2 \times 20$ min washes with 0.1 м SC. Brains were individually placed in $1 \mathrm{mg} / \mathrm{ml}$ hyperoxygenated DAB (ESM 13802) dissolved in $0.1 \mathrm{M}$ SC buffer. DAB was hyperoxygenated by bubbling medical grade $\mathrm{O}_{2}$ through solution for $2 \mathrm{~h}$ on ice before use. Brains were then exposed to strong blue light $(480 \mathrm{~nm}$ ) mercury lamp (powered by an 
ArcLamp Power Supply HBO100 DC IGN, LEP) until the DAB precipitate reaction was visible, typically $60-75 \mathrm{~min}$ at room temperature. After light exposure, brains were placed in $0.1 \mathrm{M}$ SC buffer on ice until all samples were completed.

Electron microscopy. The brain preparation for ultrastructure studies was performed as previously described (Doll et al., 2017; Vita and Broadie, 2017). All incubations were performed at room temperature with rotation unless otherwise stated. Briefly, following photoconversion, brains were postfixed in fresh $2.5 \%$ glutaraldehyde (EMS 16120) in $0.1 \mathrm{M} \mathrm{SC}$ buffer overnight at $4^{\circ} \mathrm{C}$. Brains were then washed $3 \times 20 \mathrm{~min}$ with $0.1 \mathrm{M} \mathrm{SC}$ buffer, with a final wash overnight at $4^{\circ} \mathrm{C}$. Brains were then incubated in $1 \%$ osmium tetroxide ( $\mathrm{OsO}_{4}$, EMS 19172) in $0.1 \mathrm{M} \mathrm{SC}$ buffer for $1 \mathrm{~h}$, followed by $3 \times 20 \mathrm{~min}$ washes in $0.1 \mathrm{~m} \mathrm{SC}$ buffer. Brains were then stained with $2 \%$ aqueous uranyl acetate (EMS 22400-2) covered for $2 \mathrm{~h}$, followed by $3 \times 20 \mathrm{~min}$ washes with deionized water. Brains were then dehydrated in an ethanol series (EtOH, EMS 15055): 30\%, 50\%, $70 \%, 90 \%, 95 \%, 2 \times 100 \%$ for 10 min each. EtOH was then replaced with transitional solvent propylene oxide (PO, EMS 20401): 50/50 EtOH/PO, $2 \times 100 \%$ PO each for $10 \mathrm{~min}$. Resin (20 g EMBED 812 [EMS 14900], $9 \mathrm{~g}$ DDSA [EMS 19000], 12 g NMA [EMS 13710], 1.2 g BDMA [EMS 11400]) was then infiltrated into the samples: $75 / 25 \mathrm{PO} / \mathrm{resin}$ for $30 \mathrm{~min}$, $50 / 50 \mathrm{PO} /$ resin for $1 \mathrm{~h}$, and 50/50 $\mathrm{PO} /$ resin overnight with vials open to allow PO to evaporate. Fresh resin was then applied to brains for 2, 4, and $24 \mathrm{~h}$. Flat block molds were half filled and heated at $60^{\circ} \mathrm{C}$, until resin was tacky but not yet firm $(\sim 4 \mathrm{~h})$. A small cavity was made in the resin to orient the brains anteriorly toward the block face (for sectioning in an anterior to posterior plane). Blocks were filled with resin and placed at $60^{\circ} \mathrm{C}$ for $48 \mathrm{~h}$. Thick sections (500 nm) were cut using an EM UC7 ultramicrotome (Leica Microsystems) until brain tissue was reached. After cutting $30 \mu \mathrm{m}$ of thick sections (VM7 depth based on confocal measurements), thin sections ( $65 \mathrm{~nm}$ ) were cut for $20 \mu \mathrm{m}$. Sections were collected on formvar-coated, slotted grids (EMS FCF2010-CU) at 20 sections/grid $(\sim 1.3 \mu \mathrm{m}$ of tissue/grid). Brains were imaged using a FEI $\mathrm{T}-12$ transmission electron microscope operating at $100 \mathrm{kV}$. Imaging began at $\sim 40 \mu \mathrm{m}$ and ended at $\sim 46.5 \mu \mathrm{m}$ (5 grids) to ensure no artifacts due to depth of measurement. One section per grid was imaged to limit double imaging of synaptic regions. Area measurements of labeled OSNs were made (one AL/brain) using ImageJ (Schneider et al., 2012). Synaptic active zone T-bars were quantified in two ways: (1) total within the entire section (6500× magnification) and (2) density per miniSOGlabeled Or42a OSN area.

Statistical analyses. All statistical analyses were performed with Prism software (GraphPad), except for the linear regressions done in $\mathrm{R}(\mathrm{R}$ Project for Statistical Computing, RRID:SCR_001905; version $\times 64$ 3.2.1). Statistics were done using $n=$ number of preparations, unless otherwise stated. All groups that met the criteria for parametric statistics were analyzed with unpaired two-tailed $t$ tests; otherwise, the MannWhitney test was used for comparisons. For data comparing $\geq 2$ genotypes, a two-way ANOVA was used with odorant exposure and genotype as independent variables, followed up Sidak's multiple-comparisons tests to compare the odorant vehicle and EB-exposed conditions within a genotype. To compare between two genotypes, the interaction term from the two-way ANOVA was used. For data with more than two genotypes, a linear regression was performed in $\mathrm{R}$ with interaction terms between each experimental genotype and EB exposure assessed for significance with unpaired two-tailed $t$ tests adjusted by Sidak's correction for multiple comparisons. These pairwise comparisons show how different genotypes impact the effect of EB exposure and are indicated in figures by dashed significance bars. For data comparing a genotype with $\geq 2$ odor exposure paradigms (see Fig. 3), a Kruskal-Wallis one-way ANOVA with Dunn's multiple-comparisons tests were used for pairwise comparisons between odor treatments. For analyses of synaptic T-bars per terminal area (see Fig. 5), a ROUT outlier test was done with Q set to $1 \%$.

\section{Results}

Critical period odorant experience selectively reduces OSN glomerular innervation

Previous work from our laboratory and others has shown that specific AL glomeruli exhibit changes in response to odorant ex- posure in an early-use period immediately following eclosion (Devaud et al., 2001, 2003a,b; Sachse et al., 2007; S. Das et al., 2011; Acebes et al., 2012; Sudhakaran et al., 2014; Doll and Broadie, 2015, 2016). Older work has shown that this early sensory experience can result in expansion of OSN innervation of the activated glomeruli (Sachse et al., 2007; S. Das et al., 2011). We started this study by testing this result for Or42a OSNs innervating the VM7 glomerulus, which are strongly activated by the EB odorant (Münch and Galizia, 2016). To examine the VM7 innervation, we labeled the OSNs with an Or42a-Gal4 driving UASmCD8::GFP to mark neural membranes (Fig. 1). Or42a OSN innervation of the VM7 glomerulus was compared between an odorant vehicle control (mineral oil) and $\mathrm{EB}$ dissolved in mineral oil at low (15\% EB; Fig. 1A) and high (25\% EB; Fig. 1B) concentrations. Odorant exposure was compared between an early time period (0-2 d post eclosion [dpe]; Fig. $1 A, B)$ within the welldefined early-use critical period (Devaud et al., 2003a; Sachse et al., 2007; Tessier and Broadie, 2008, 2011; Doll and Broadie, 2015, 2016; Doll et al., 2017; Vita and Broadie, 2017; Sears and Broadie, 2018) and a late time period of adult maturity following the critical period (7-9 d dpe; Fig. 1C,D). Sample images at both low and high magnification, as well as quantified glomerular volume data for all eight conditions, are shown in Figure 1.

During the critical period, EB odorant exposure causes dramatic reduction in Or42a OSN innervation of VM7 glomeruli (Fig. 1 $A, B$ ). With 15\% EB, VM7 innervation is strongly reduced compared with vehicle control (oil alone), with weak and sparse Or42a OSN labeling in the VM7 synaptic domain (Fig. 1A, left two panels). Despite a strong reduction in innervation, glomerulus integrity is not compromised, with similar glomerular boundaries and maintained scaffolding. Quantification of the innervation volume shows that EB-exposed animals are significantly reduced: normalized vehicle control (oil) $1.0 \pm 0.066(n=$ 9 brains $)$ versus $15 \%$ EB $0.429 \pm 0.116(n=10)\left(t_{(17)}=4.148\right.$, $p=0.0007$, two-tailed unpaired $t$ test; Fig. $1 A$, right). The EB exposure effect is concentration-dependent, with $25 \%$ EB causing an even greater loss of VM7 innervation, often resulting in an absence of detectable Or42a OSNs (Fig. 1B, left). The loss of VM7 innervation is accompanied by loss from the labial tract, which projects from the maxillary palps to AL. Quantification of glomerular volume reveals that EB-exposed animals show a greater and more significant loss of VM7 innervation compared with matched controls: oil $1.0 \pm 0.037(n=23)$ versus $25 \% \mathrm{~EB}$ $0.195 \pm 0.054(n=26)\left(t_{(47)}=11.95, p=7.53 \times 10^{-16}\right.$, twotailed unpaired $t$ test; Fig. $1 B$, right). Or42a OSN remodeling after $25 \% \mathrm{~EB}$ can appear qualitatively similar to the $15 \%$ EB condition, but there is a greater extent of axon process retraction, including many instances of complete loss of detectable Or42a OSN signal (Fig. 1B). These results show that EB odorant during the early-use critical period ( $0-2 \mathrm{dpe}$ ) exposure causes a dose-dependent reduction of Or42a OSN innervation of the VM7 glomerulus.

At maturity, EB odorant exposure causes little or no change in Or42a OSN innervation of VM7 glomeruli (Fig. 1C,D). With $15 \%$ EB for $2 \mathrm{~d}$ starting at $7 \mathrm{dpe}$, there is no significant difference in VM7 innervation compared with controls (Fig. 1C). The size and structure of Or42a OSN axon tracts entering the VM7 glomerulus (Fig. $1 C$, left), and the VM7 innervation by Or42a terminals (Fig. $1 C$, middle), both appear unchanged between $\mathrm{EB}$ exposure and vehicle controls. Quantification of innervation volume shows that EB-exposed animals are not significantly different from controls: oil $1.0 \pm 0.055(n=20)$ versus $15 \% \mathrm{~EB}$ $0.841 \pm 0.066(n=18)\left(t_{(36)}=1.862, p=0.071\right.$, two-tailed unpaired $t$ test; Fig. $1 C$, right). With the higher $25 \%$ EB exposure 

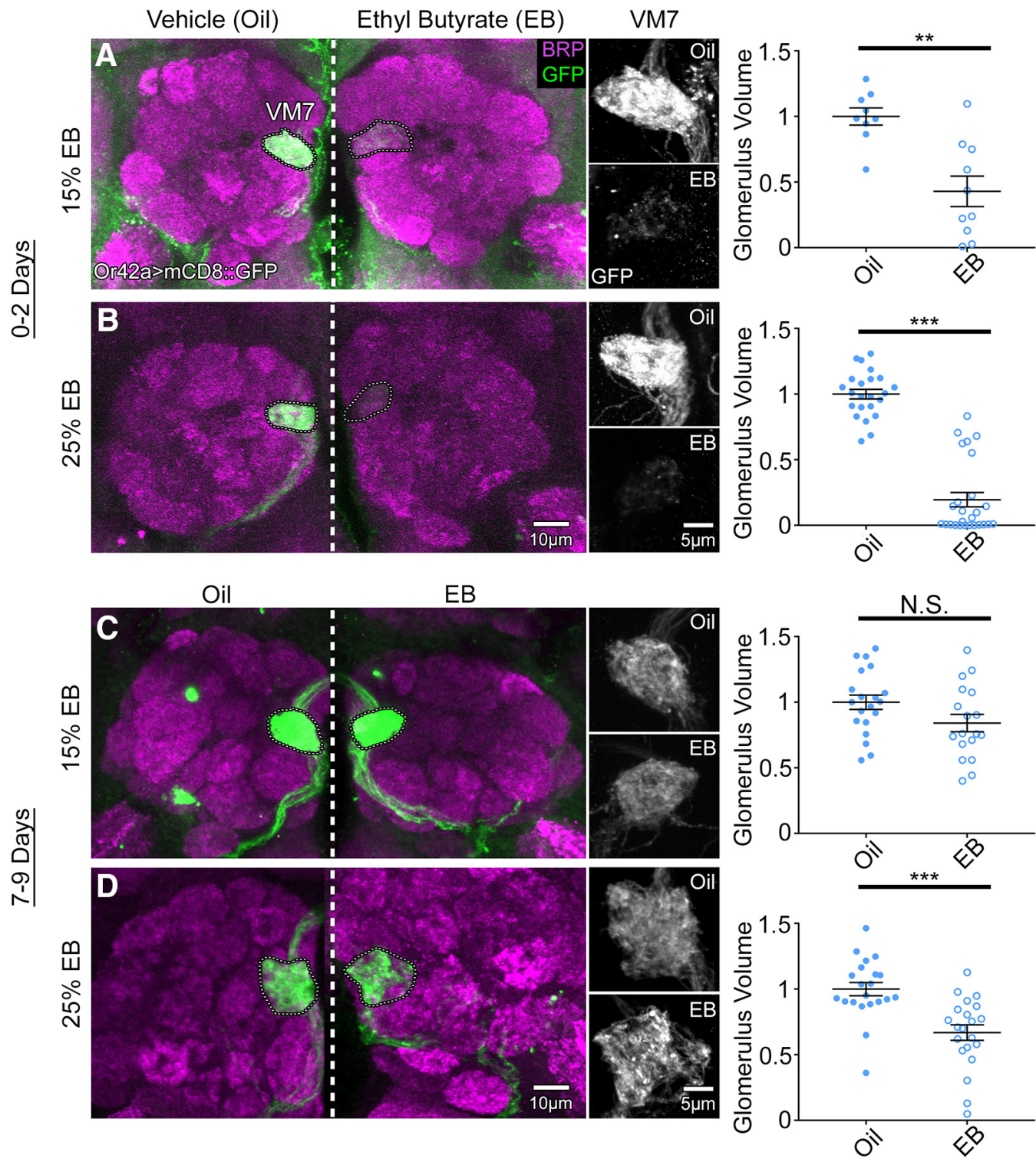

Figure 1. Critical period-sensitive remodeling of olfactory glomeruli innervation. Representative confocal images of the Or42a-Gal4-driven UAS-mCD8::GFP membrane marker (green) in 0SNs innervating AL glomerulus VM7 double-labeled with the anti-BRP (magenta) synaptic marker. Animals exposed to mineral oil vehicle (left) or EB odorant in oil during the early-use critical period (0-2 dpe) at $(\boldsymbol{A}) 15 \%$ or $(\boldsymbol{B}) 25 \%$ (\% v/v; right). Middle, High-magnification grayscale images of the Or42a-GFP channel only for the oil vehicle (top) and EB (bottom) at both odorant concentrations. Right, Quantification of the Or42a OSN innervation volume of the VM7 glomerulus for 15\% (top) and 25\% (bottom) odorant concentrations. The same experiment at maturity (7-9 dpe) comparing oil vehicle (left) with EB in oil at (C) 15\% or (D) 25\%. Middle, High-magnification grayscale images of GFP channel only. Right, Quantification of Or42a 0SN innervation volume of the VM7 glomerulus at both odorant concentrations. Scatter plots represent all data points and the mean \pm SEM. Significance from two-tailed unpaired $t$ test is indicated. Not significant $(p>0.05)$. ${ }^{* *} p<0.01$. ${ }^{* * *} p<0.001$.

(7-9 dpe), there is weak Or42a OSN remodeling (Fig. 1D). Robust VM7 glomerulus innervation persists, although there is evidence of some axon retraction and glomerular reduction (Fig. $1 D$, left, middle). Quantification shows a significant loss of the VM7 glomerular volume: oil $1.0 \pm 0.051(n=21)$ versus $25 \% \mathrm{~EB}$ $0.669 \pm 0.059(n=21)\left(t_{(40)}=4.262, p=0.0001\right.$, two-tailed unpaired $t$ test; Fig. $1 D$, right). However, this change is small compared with critical period remodeling $(\sim 80 \%$ reduction in critical period vs $\sim 30 \%$ at maturity). This result is consistent with mammalian critical periods, in which heightened sensory stimulation can induce adult plasticity after critical period closure (Hensch and Bilimoria, 2012; Baroncelli et al., 2016). These results show that EB odorant exposure drives remodeling loss of Or42a OSN innervation in the VM7 glomeruli, which is concentration-dependent and especially sensitive during the critical period (0-2 dpe).

\section{Critical period odorant exposure does not alter OSN survival} or odorant receptor expression

The dramatic loss of Or42a OSN innervation of the VM7 glomerulus after critical period EB exposure could be the result of the OSN cell death or reduced expression of the cell marker. However, studies of AL glomerular innervation after odorant exposure or neuronal silencing have reported no alterations of OSN cell bodies or peripheral odor responses, regardless of the direction of the glomerular volume change (Devaud et al., 2001; Sachse et al., 2007; Chiang et al., 2009). Moreover, despite the widespread use of genetically encoded odorant receptor fluores- 
A
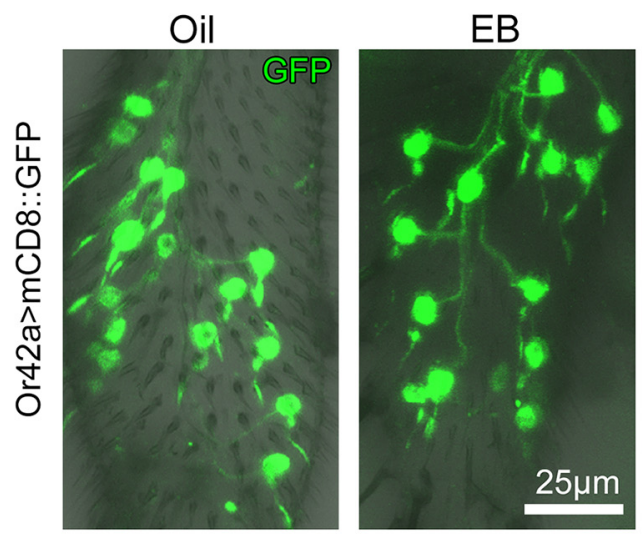

C

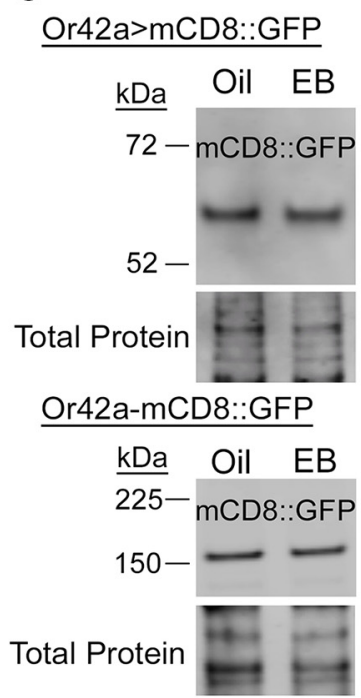

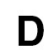
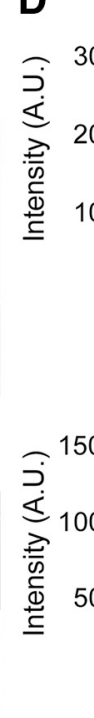

B
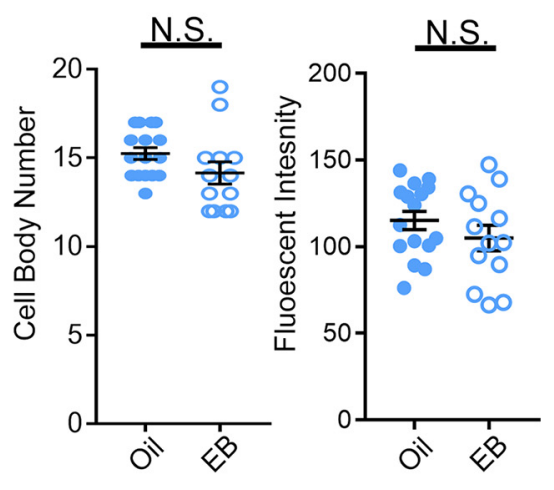

E

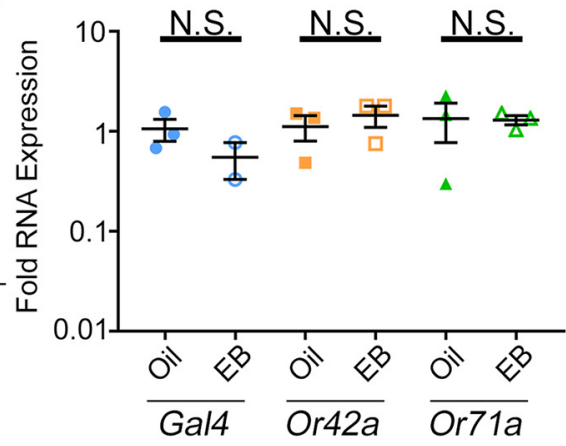

Figure 2. Odorant experience does not alter OSN survival or odorant receptor expression. $A$, Representative confocal images of Or42a-Gal4-driven UAS-mCD8::GFP membrane marker (green) in the OSN cell bodies, dendrites, and labial tract within the maxillary palp. Staged animals exposed to either the mineral oil vehicle (oil, left) or odorant EB in oil ( $25 \% \mathrm{v} / \mathrm{v}$, right) during the critical period (0-2 dpe). B, Quantification of the number of Or42a OSN cell bodies (left) and total fluorescent intensity within each maxillary palp (right) comparing oil and EB conditions. $\boldsymbol{C}$, Representative Western blots of maxillary palp homogenates from oil or EB-exposed animals (0-2 dpe) from both Or42a-Gal4-driven mCD8::GFP (0r42a > mCD8::GFP, top) and mCD8::GFP driven directly by a 0r42a promoter (Or42a-mCD8::GFP, bottom). Both blots show the GFP channel (top) and total protein (REVERT, bottom). Left, Molecular weights. The difference in mCD8::GFP size is due to the number of GFP molecules in reporter (1 GFP at top vs 4 GFPs at bottom). D, Quantification of GFP Western blots for both Or42a-Gal4-driven mCD8::GFP (top) and 0r42a promoter (bottom) normalized to the total protein. A sample size of three independent biological replicates is compared for all four conditions. $\boldsymbol{E}$, Quantification of RNA level from the proboscis with attached maxillary palps from animals exposed to oil or EB from 0 to 2 dpe for the Gal4 driver (left), Or42a receptor (middle), and Or71a (EB-insensitive control olfactory receptor, right) normalized to the GAPDH2 housekeeping gene. Scatter plots represent all data points and the mean \pm SEM. $\boldsymbol{B}$, Significance from two-tailed unpaired $t$ tests. $\boldsymbol{D}, \boldsymbol{E}$, Significance from Mann-Whitney tests. Not significant $(p>0.05)$.

cent reporters to measure AL glomerulus innervation volumes, no work to our knowledge has shown altered transgenic expression after odorant exposure (Sachse et al., 2007; S. Das et al., 2011; Acebes et al., 2012). Nevertheless, to test for any altered OSN survival after EB exposure during the critical period, Or42a OSN cell bodies located in the peripheral olfactory maxillary palp were quantified. As above, animals with Or42a-Gal4 driving UASmCD8::GFP were exposed to $25 \%$ EB or vehicle control for the first $2 \mathrm{~d}$ after eclosion. Critical period odor exposure does not alter Or42a OSN cell bodies (Fig. 2A). Quantification shows no significant difference in soma number: oil $15.25 \pm 0.335$ cells/ palp $(n=16)$ versus EB $14.15 \pm 0.629(n=13)\left(t_{(27)}=1.62, p=\right.$ 0.117 ; Fig. $2 B$ ). Likewise, critical period odor exposure does not alter Or42a-Gal4-driven GFP expression in OSN cell bodies (Fig. $2 A)$. Quantification shows no significant difference in fluorescence intensity of soma and basal processes: oil 115.2 \pm 5.261 $(n=16)$ versus $\mathrm{EB} 105.1 \pm 7.37(n=13)\left(t_{(27)}=1.143, p=\right.$ 0.263; two-tailed unpaired $t$ tests; Fig. $2 B)$.
To confirm and extend the above findings, we next used Western blot analyses to test maxillary palps for differences in Or42aGal4-driven UAS-mCD8::GFP expression between the same paired EB odorant exposed and oil vehicle control conditions. In agreement with the above immunocytochemistry imaging, Western blot analyses also do not show GFP expression changes after critical period EB exposure assayed in three independent replicates (Fig. 2C, top). Quantification of the fluorescence intensity normalized to total protein reveals no significant difference between the two conditions: oil $1533 \pm 192.3(n=3$ replicates, $>30$ palps/replicate) versus EB $1900 \pm 606.9(n=3)(U=3, p=0.7$; two-tailed Mann-Whitney test; Fig. 2D, top). We also separately assayed mCD8::GFP expression under the direct control of the Or42a promoter (Or42a-mCD8::GFP), which provides a direct, Gal4-indendent read-out of Or42a expression (Stephan et al., 2012). Western blot analyses were done to test GFP expression changes after critical period EB exposure in three independent replicates. Maxillary palps from these animals also show no 
change in GFP expression following critical period EB exposure (Fig. 2C, bottom). Quantification of fluorescence intensity normalized to total protein again reveals no significant difference between the two conditions: oil $4735 \pm 1008(n=3$ replicates, $>20$ palps/replicate $)$ versus EB $6561 \pm 1722(n=3)(U=4, p>$ 0.999; two-tailed Mann-Whitney test; Fig. 2D, bottom). Thus, both imaging and Western blot analyses indicate no change in Or42a-driven expression in response to heightened EB odorant exposure during the critical period.

Finally, we used qRT-PCR to directly test transcript levels following critical period EB exposure. After either oil vehicle or $25 \%$ EB exposure ( $0-2 \mathrm{~d}$ ), proboscises with maxillary palps were dissected to extract RNA. We assessed the expression of three transcripts as follows: (1) Gal4, (2) the Or42a receptor, and (3) an Or71a receptor control, all normalized to the housekeeping genes GAPDH2 and rp49. Compared with GAPDH2, quantification of RNA expression levels between EB-exposed animals and oil vehicle controls done using the $\Delta \Delta$ Ct method show no significant differences (Gal4: oil $1.061 \pm 0.262$ fold expression, $n=3$ replicates, 10 proboscises for all replicates, vs EB $0.551 \pm 0.221, n=2$, $U=1, p=0.4$; Or42a: oil $1.12 \pm 0.32, n=3$, vs EB $1.45 \pm 0.348$, $n=3, U=2, p=0.4$; Or71a: oil $1.348 \pm 0.574, n=3$, vs EB $1.302 \pm 0.14, n=3, U=4, p>0.999$; two-tailed Mann-Whitney test; Fig. 2E). Compared with $r p 49$, a second housekeeping gene, there are likewise no significant differences in RNA levels (Gal4: oil $1.071 \pm 0.293$ fold expression, $n=3$ replicates, 10 proboscises for all replicates, vs EB $0.362 \pm 0.036, n=2, U=0, p=0.2$; Or42a: oil 1.163 $\pm 0.371, n=3$, vs EB $1.147 \pm 0.512, n=3, U=$ 4, $p>0.999$; Or71a: oil 1.415 $\pm 0.623, n=3$, vs EB 1.037 \pm 0.396 , $n=3, U=4, p>0.999$; two-tailed Mann-Whitney test). These results demonstrate that the dramatic loss of Or42a OSN innervation of the VM7 glomeruli following heightened critical period odorant experience is independent of changes in OSN cell bodies, Or42a reporter expression, or Or42a receptor level, and therefore due to local retraction of axon terminal innervation in the AL.

\section{Retracted OSN glomerular innervation during critical period odorant exposure is reversible}

Given the striking OSN axonal retraction caused by heightened odorant experience during the critical period (Fig. 1), we next wished to test whether removal of the odorant stimulus during this time window would enable reinnervation of the VM7 glomerulus by Or42a OSNs. Previous studies investigating the specialized $\mathrm{CO}_{2}$-sensitive OSN showed that odorant-dependent glomerular growth is reversible after $\mathrm{CO}_{2}$ removal for at least $2 \mathrm{~d}$ during the critical period, but not at all reversible afterward (Sachse et al., 2007). A similar critical period-restricted reversibility characterizes the mammalian ocular dominance activitydependent critical period (Blakemore et al., 1978). We therefore hypothesized that the removal of the EB odorant after inducing critical period remodeling would enable Or42a OSNs to reinnervate VM7 glomeruli, thus reversing the effect during the critical period. Using OSNs labeled with Or42a-Gal4 driving UASmCD8::GFP, we tested the reversibility of OSN axonal retraction by shifting animals after 1 or $2 \mathrm{~d}$ of EB odorant exposure immediately after eclosion, to oil through $4 \mathrm{dpe}$ (Fig. 3A). We tested the effects of EB exposure for 1, 2, and $4 \mathrm{~d}$, demonstrating a similar strong axon retraction for all three critical period treatments (Fig. $3 A, B)$. In parallel, we tested animals exposed to EB for $1 \mathrm{~d}(0-1$ dpe) and then returned to the oil vehicle for the 3 remaining days ( $3 \mathrm{~d}$ reversal; Fig. $3 A$ ), as well as animals exposed to EB for $2 \mathrm{~d}$ ( $0-2$ dpe) replaced with the oil vehicle for the 2 remaining days ( 2 $\mathrm{d}$ reversal; Fig. $3 A$ ). All animals were dissected at the same time at the end of day 4 (Fig. 3A), and the Or42a OSN innervation of the VM7 glomerulus imaged (Fig. $3 B, C$ ).

EB exposure for 1, 2, or $4 \mathrm{~d}$ during the critical period causes a similar reduction in the VM7 innervation compared with the oil controls (Fig. $3 B, D$; Kruskal-Wallis one-way ANOVA $\mathrm{KW}_{(7)}=$ $84.94, p=1.348 \times 10^{-15}$ ). The dimmer Or42a $>$ GFP signal at 1 dpe reflects the earlier time point in reporter expression, but the extent of axonal retraction is similar for all three treatments. Quantification shows that all EB exposures significantly reduce glomerular volume compared with oil controls (1 d: oil $1.184 \pm$ $0.047, n=26$, vs EB $0.571 \pm 0.073, n=23$, mean rank difference $=80.82, p=2.21 \times 10^{-6} ; 2 \mathrm{~d}$ : oil $1.303 \pm 0.053, n=22$, vs EB $0.646 \pm 0.093, n=26$, mean rank difference $=82.33, p=$ $1.79 \times 10^{-6}$; 4 d: oil $1.0 \pm 0.040, n=23$, vs EB $0.505 \pm 0.070, n=$ 24 , mean rank difference $=58.32, p=0.0017$, Dunn's multiplecomparisons tests; Fig. 3D). We find that both the 2 and $3 \mathrm{~d}$ reversal returns innervation to levels indistinguishable from controls (Fig. 3C). Quantification shows that animals returned to the oil vehicle for 2 or $3 \mathrm{~d}$ during the critical period redevelop OSN innervation volumes that are not statistically different from day 4 controls never exposed to the EB odorant ( $3 \mathrm{~d}$ reversal $1.164 \pm$ $0.044, n=25$, mean rank difference $=-0.3, p>0.9999 ; 2 \mathrm{~d}$ reversal $1.01 \pm 0.087, n=24$, mean rank difference $=-27.82$, $p=0.424$, Dunn's multiple-comparisons tests; Fig. 3D). In addition to the reversal of the majority of animals after $2 \mathrm{~d}$ of $\mathrm{EB}$ exposure, 3 animals fail to reverse the axonal retraction (Fig. 3D), suggesting the approaching end of the early-use critical period. We conclude the Or42a OSN innervation remodeling within the VM7 glomerulus is reversible after removal from EB odor during the critical period.

\section{Critical period odorant exposure drives OSN synapse elimination in AL glomeruli}

The glomerular OSN volume measure represents a good proxy for synaptic innervation (Mosca and Luo, 2014) but does not directly measure OSN synaptic contacts. We therefore tested whether the Or42a OSN volume reduction in response to EB odorant exposure during the early-use critical period also affects synaptic organization within the VM7 glomerulus, by assaying the commonly used active zone marker BRP (Wagh et al., 2006). Based on the reduction in glomerular volume, we hypothesized that synapse number would be reduced. We used the Synaptic Tagging with Recombination (STaR) labeling method (Chen et al., 2014) to investigate the Or42a OSN-specific changes in BRP expression and spatial distribution in synapses following critical period odorant exposure. The STaR method uses the transgenic FLP/FRT system to express tagged BRP::GFP under its endogenous promoter only in the neurons where FLP is expressed. Here, we use the UAS/Gal4 system to specifically express FLP using Or42a-Gal4 targeted to the OSNs innervating only the VM7 glomerulus. The resulting brains show green, punctate BRP::GFP synaptic labeling restricted to the mDC8::RFP-colabeled VM7 glomerulus (Fig. 4). The transgenic BRP::GFP label shows close overlap with anti-BRP staining for endogenous VM7 synapses. Or42a OSN synapses are compared between the odorant vehicle control (mineral oil) and 25\% EB dissolved in the oil during the early-use critical period ( $0-2$ dpe). Sample images and quantified data for VM7 glomerular volume, BRP punctae number, and BRP expression levels are shown in Figure 4.

Vehicle control animals exhibit prominent BRP::GFP synaptic labeling of Or42a OSN presynaptic active zones in the VM7 glomerulus (Fig. $4 A$, top). In contrast, animals exposed to $25 \%$ 
A

\begin{tabular}{|c|c|c|c|c|c|}
\hline Condition & Day 1 & Day 2 & Day 3 & Day 4 & \\
\hline Day Reversal & EB & Oil & Oil & Oil & Dissect \\
\hline Rau Pouna & EB & EB & Oil & Oil & Dissect \\
\hline
\end{tabular}

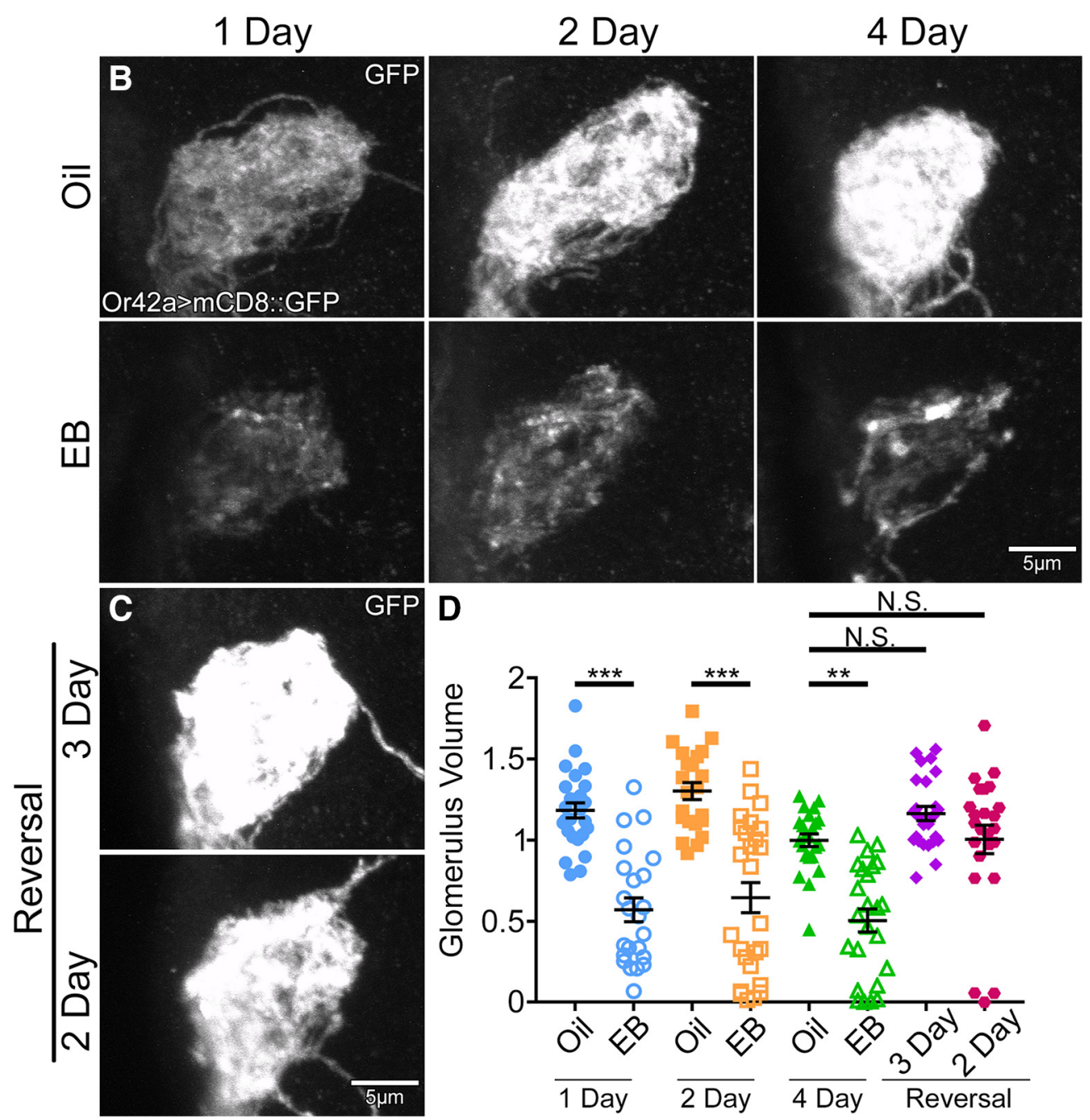

Figure 3. Critical period OSN innervation remodeling is reversible by odorant removal. $\boldsymbol{A}$, Schematic illustrating the timing of exposure to either mineral oil vehicle (oil) or EB in oil (25\%) for reversal experiments. Animals were exposed to EB for either $1 \mathrm{~d}$ (top) or $2 \mathrm{~d}$ (bottom) before shifting to the oil vehicle condition. All animals were dissected at the end of $4 \mathrm{~d}$, testing $3 \mathrm{~d}$ reversal (top), or $4 \mathrm{~d}$ reversal (bottom). $\boldsymbol{B}$, Representative grayscale confocal images of Or42a-Gal4-driven UAS-mCD8::GFP membrane marker in OSNs innervating the AL glomerulus VM7. Animals exposed to either oil vehicle (top) or EB (bottom) for $1 \mathrm{dpe}$ (left), $2 \mathrm{dpe}$ (middle), and $4 \mathrm{dpe}$ (right). C, Representative grayscale images as in $\boldsymbol{B}$, but with $1 \mathrm{~d}$ of $25 \%$ EB exposure followed by $3 \mathrm{~d}$ of oil ( $3 \mathrm{~d}$ reversal, top) or $2 \mathrm{~d}$ of EB exposure followed by $2 \mathrm{~d}$ of oil ( $2 \mathrm{~d}$ reversal, bottom). $D$, Quantification of Or42a 0SN innervation volume of the VM7 glomerulus for all 8 conditions. Scatter plots represent all data points and the mean \pm SEM. Kruskal-Wallis one-way ANOVA with follow-up Dunn's multiple-comparisons tests: not significant $(p>0.05) ;{ }^{* *} p<0.01 ;{ }^{* *} p<0.001$.

EB during the critical period (0-2 dpe) show a striking decrease in both the number and intensity of BRP::GFP-labeled synaptic punctae (Fig. $4 A$, bottom). As previously shown, Or42a OSN innervation of VM7 in EB-exposed animals is significantly reduced compared with controls (oil $1.0 \pm 0.0368, n=16$, vs EB $0.332 \pm 0.0898, n=12 ; t_{(26)}=7.566, p=4.953 \times 10^{-8}$, twotailed unpaired $t$ test; Fig. $4 B$ ). Consistently, EB exposure also causes a highly significant reduction of local BRP signal maxima as a measure for BRP synaptic punctae (see Materials and Methods) compared with matched controls (oil $1.0 \pm 0.048, n=14$, vs EB $0.241 \pm 0.068, n=12 ; t_{(24)}=9.292, p=2.0217 \times 10^{-9}$, two-tailed unpaired $t$ test; Fig. $4 C$ ). Similarly, the overall BRP signal intensity within the VM7 glomerulus is reduced by EB exposure during the critical period compared with vehicle controls (oil $1.0 \pm 0.0596, n=14$, vs EB $0.179 \pm 0.103, n=12 ; U=$ $13, p=7.704 \times 10^{-5}$, two-tailed Mann-Whitney test; Fig. $\left.4 D\right)$. These results indicate that critical period olfactory experience induces OSN synapse elimination, with fewer synapses persisting after the odorant exposure. The greater BRP reduction compared with OSN glomerulus volume suggests that synapse loss precedes retraction of Or42a axons. Together, these results show that $\mathrm{EB}$ odorant exposure during the critical period drives rapid synapse elimination from the Or42a OSNs innervating the VM7 glomerulus. 

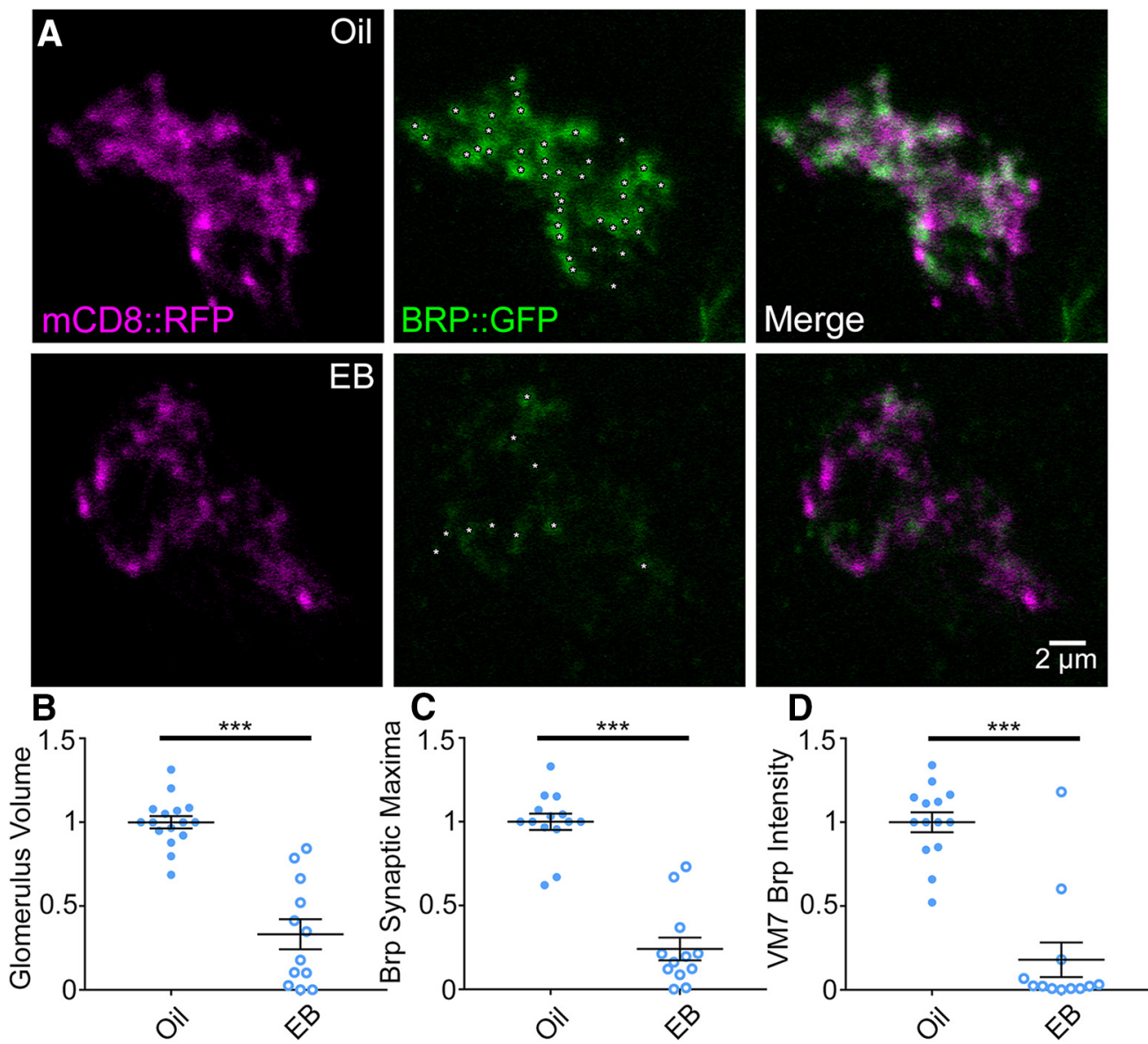

Figure 4. Critical period olfactory experience drives glomeruli synapse elimination. $A$, Representative confocal images of Or42a-Gal4-driven UAS-mCD8::RFP membrane marker (mCD8::RFP, magenta, left) and UAS-Bruchpilot::GFP (BRP::GFP, green, middle) with the merged overlap (right) in OSNs innervating the AL glomerulus VM7. BRP local maxima punctae at OSN synapses (white asterisks) in single confocal sections $(1 \mu \mathrm{m})$. Exposure to the mineral oil vehicle (top) or EB odorant at $25 \%(\% \mathrm{v} / \mathrm{v}$; bottom) during the early-use critical period ( $0-2 \mathrm{dpe})$. Quantification of the $0 \mathrm{r} 42 \mathrm{a}$ OSN innervation volume of the VM7 glomerulus $(\boldsymbol{B})$, BRP synaptic maxima $\left(\boldsymbol{C}\right.$, and BRP intensity $(\boldsymbol{D})$. Scatter plots represent all the data points and the mean \pm SEM. ${ }^{* * *} p<0.001$ (two-tailed unpaired $t$ tests).

\section{Ultrastructural analyses of odorant exposure effects on Or42a OSN innervation}

We next sought to test and extend the above light microscopy study at higher resolution using transmission electron microscopy (TEM) ultrastructural analyses (Vita and Broadie, 2017). The AL VM7 glomerulus contains multiple neuron types in addition to Or42a OSNs (Golovin and Broadie, 2016, 2017; Grabe et al., 2016), making it impossible to identify specific synaptic terminals using standard TEM sample preparations. Therefore, to specifically assay Or42a OSN synaptic terminals within the VM7 glomerulus, we made use of a photoconversion labeling technique to render the Or42a OSNs electron-dense compared with all other surrounding VM7 neurons. The genetically encoded mini Singlet Oxygen Generator (miniSOG) construct produces oxygen free radicals when exposed to blue light $(480 \mathrm{~nm})$, which can then interact with DAB to generate a dark, electrondense precipitate at the location of the reaction (Shu et al., 2011; $\mathrm{Ng}$ et al., 2016). This signal can be readily imaged with TEM. Driving targeted expression of the membrane-tethered mCherrytagged miniSOG transgene (UAS-myr-miniSOG-mCherry) with Or42a-Gal4, we can label and identify specifically the Or42a OSN synaptic terminals innervating the VM7 glomerulus. Before the blue light exposure, Or42a OSNs expressing miniSOG are clearly visible and identifiable via the fluorescent mCherry tag (Fig. $5 \mathrm{~A}$, left, red). After the blue light exposure $(\sim 1 \mathrm{~h})$, the DAB precipitate labels the Or42a OSNs innervating only the VM7 glomerulus
(Fig. $5 \mathrm{~A}$, right, black), marking these neurons selectively for subsequent TEM analyses.

Because of the shape and location of the VM7 glomerulus, proper brain orientation in the TEM block is imperative to maintain imaging consistency (Fig. 5B). To section the embedded brain, we position the anterior side toward the block face, such that sectional planes proceed in an anterior to posterior direction (Fig. 5B). Moreover, to ensure accurate and consistent depth measurements across compared conditions, we use brain confocal imaging stacks as a guide to determine the depth from the $\mathrm{AL}$ surface to the widest point of the VM7 glomerulus $(\sim 40 \mu \mathrm{m})$. Starting at this sectioning depth, we image individual ultrathin brain sections $(65 \mathrm{~nm})$ spanning $6.5 \mu \mathrm{m}$ of tissue, affording us reproducible image acquisition and analysis (Fig. $5 B$ ). During the DAB photoconversion, the visible fluorescence signal will bleach swiftly (typically $5-10$ min of light exposure), but singlet oxygens are still being generated and blue light exposure must be maintained until the precipitate is clearly seen $(\sim 1 \mathrm{~h}$ for our experiments). Labeled Or42a OSNs are distinctly darker than all surrounding cells and can be easily identified even at low magnification $(6500 \times)$ as islets of increased density within VM7 glomeruli (Fig. 5C). In control brains, labeled regions are present throughout the VM7 glomerulus, occupying a large proportion of the neuropil (Fig. 5C, left). Under identical acquisition and imaging parameters, EB-exposed animals display a dramatic reduction in labeled Or42a OSNs, with islets sparsely occupying the 

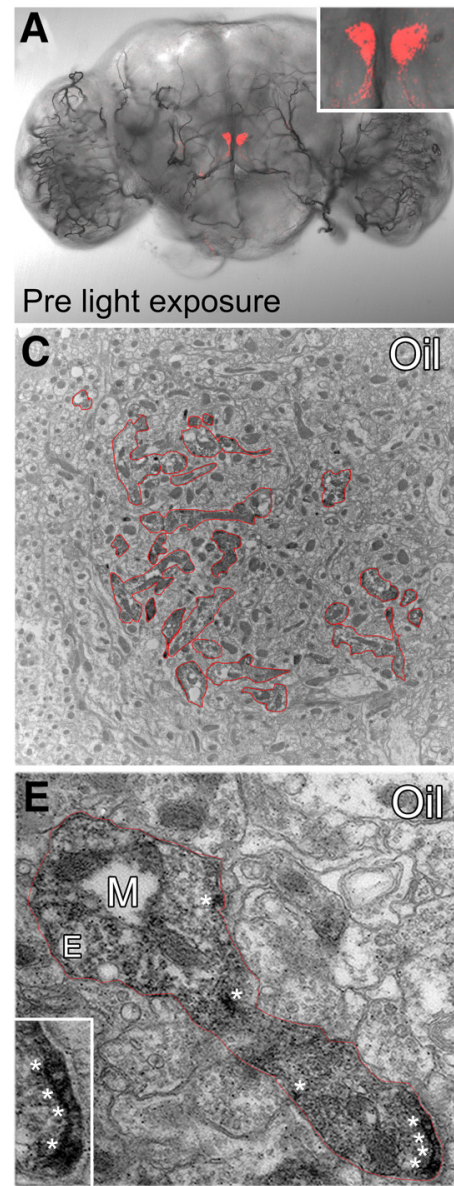
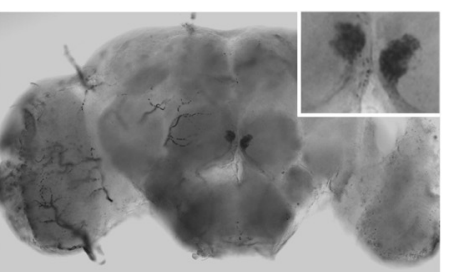

$5 \overline{0 u m}$
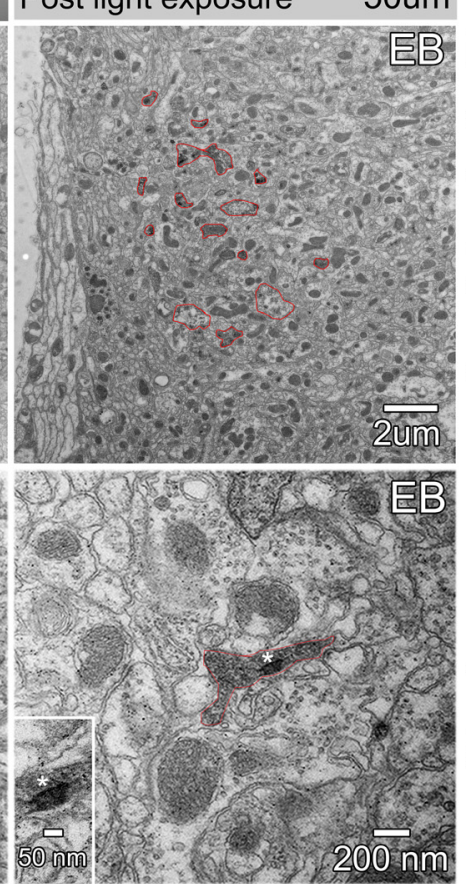

B

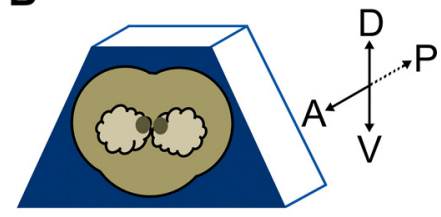

Sectional planes: $\mathrm{A} \longrightarrow \mathrm{P}$
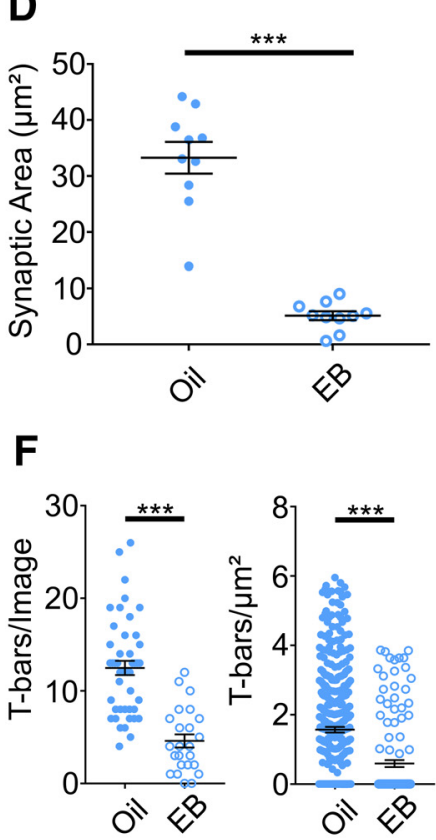

Figure 5. Ultrastructure analysis of critical period odorant exposure on Or42a innervation. $\boldsymbol{A}$, Light microscope brain images of membrane-tethered mCherry-tagged miniSOG transgene (UAS-myr-miniSOG-mCherry) driven by Or42-Gal4 before blue light ( $480 \mathrm{~nm}$ ) exposure (left) and after light exposure forming DAB precipitate (right). Insets, Enlarged VM7 glomerulus with fluorescent signal (red, left) and DAB precipitate (black, right). B, Schematic depicting brain orientation in resin block for sectioning, with the following coordinates: anterior (A), posterior (P), dorsal (D), and ventral (V). Direction of sectional plane is A to P. C, Representative low-magnification electron micrographs (6500 X) of vehicle control (oil, left) and $48 \mathrm{~h}$ EB exposure (right). Red outlines represent DAB-labeled regions in the VM7 glomerulus. $\boldsymbol{D}$, Quantification of area measurements depicted in $\boldsymbol{C}$. $\boldsymbol{E}$, Representative high-magnification electron micrographs (30,000 $\times$ ) of vehicle control (oil, left) and $48 \mathrm{~h} \mathrm{~EB}$ exposure (right). Red outlines represent DAB-labeled boutons in VM7 glomerulus. M, Mitochondrion; E, endosome. *T-bars. Insets, Enlarged synaptic T-bars from each bouton. $F$, Quantification of synaptic active zone T-bar count per image (left) and density within labeled area (right). All experiments use Or42a-Gal4-driven myr-miniSOG-mCherry. Scatter plots represent all data points and mean \pm SEM. Significance is shown between conditions by two-tailed unpaired $t$ tests or a Mann-Whitney test (T-bars/area). Analysis of T-bars per area was performed following ROUT outlier test with $Q$ set to $1 \%{ }^{* * *} p<0.001$.

neuropil (Fig. 5C, right). Thus, odorant experience during the critical period results in a dramatic loss of Or42a OSN innervation of the VM7 glomerulus.

Quantification shows a striking decrease in OSN synaptic area following 25\% EB critical period treatment (0-2 dpe) compared with vehicle controls (Fig. $5 D$ ). Vehicle controls exhibit $6 \times$ more terminal area per section compared with EB-treated animals: oil $33.280 \pm 2.839 \mu \mathrm{m}^{2}(n=10$ sections $)$ versus EB $5.103 \pm 0.800$ $\mu \mathrm{m}^{2}(n=10)$, with a highly significant reduction after the odorant exposure $\left(t_{(18)}=9.554, p<0.0001\right.$, two-tailed unpaired $t$ test; Fig. 2D). Higher magnification reveals DAB precipitate throughout the cytoplasm $(30,000 \times$; Fig. $5 E)$, consistent with all published miniSOG labeling results (Shu et al., 2011; Ng et al., 2016), likely due to membrane trafficking within the neuron (Winkle and Gupton, 2016). Synaptic regions characterized by presynaptic active zone T-bars (Fig. $5 E$, asterisks; enlarged in insets), with mitochondria $(\mathrm{M})$, endosomes (E), and synaptic vesicles, are clearly apparent in the Or42a OSN terminals innervating VM7. To test for synapse changes, we quantified T-bars in two ways: (1) T-bars per EM image and (2) T-bars density. In both cases, there is a large decrease in synaptic T-bars (asterisks) after critical pe- riod $\mathrm{EB}$ exposure (Fig. $5 E, F$ ). In both counts, oil vehicle controls contain significantly more T-bars than EB-treated animals (oil $12.480 \pm 0.763 \mathrm{~T}$-bars/image, $n=46$ images, vs EB $4.583 \pm 0.704$ T-bars/image, $n=24$ images; oil $1.57 \pm 0.082 \mathrm{~T}$-bars/ $\mu \mathrm{m}^{2}, n=$ 400 regions, vs EB $0.591 \pm 0.100 \mathrm{~T}$-bars $/ \mu \mathrm{m}^{2}, n=136$ regions; Fig. $5 F$ ). Together, these results indicate that axonal retraction and synapse elimination in the Or42a OSNs innervating the VM7 glomerulus follow EB odorant sensory experience during the critical period.

\section{Or42a olfactory reception is required for critical period remodeling of Or42a OSN innervation}

In addition to the strong activation of Or42a OSNs innervating only the VM7 glomerulus, EB can activate several other AL glomeruli, particularly with higher odorant concentrations (Couto et al., 2005; Semmelhack and Wang, 2009; Münch and Galizia, 2016). This is a very important consideration because AL lateral connections from other activated olfactory sensory neurons could possibly affect VM7 critical period development. Indeed, recent work has demonstrated that altered lateral inhibition in the AL can modulate olfactory sensory processing and change 
A

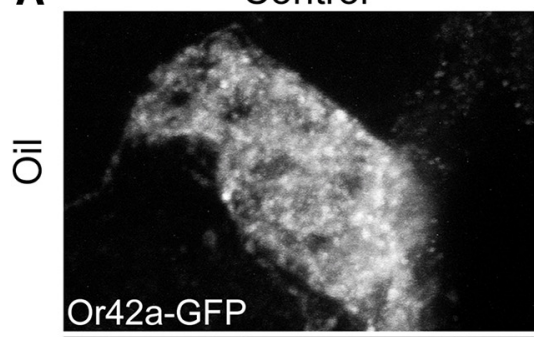

Control

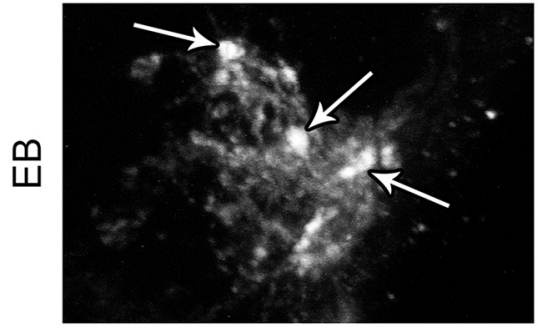

B

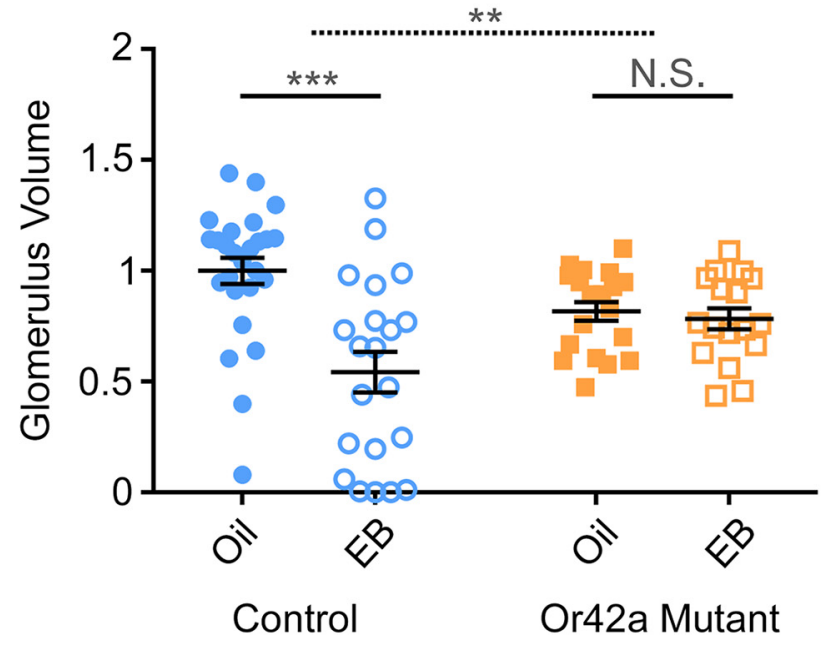

Or42a Mutant
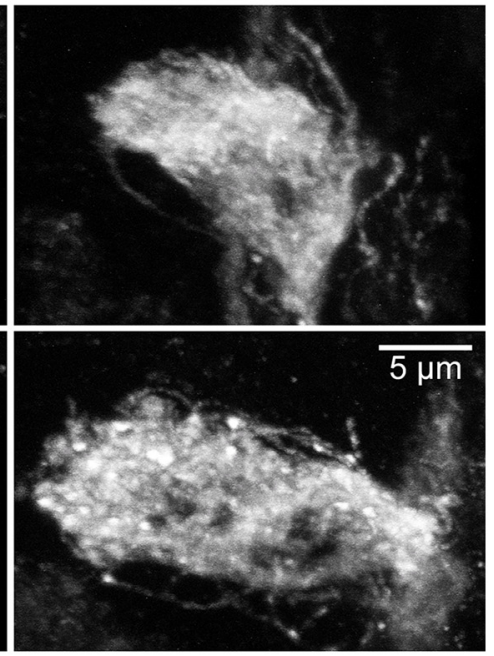

$* *$

Figure 6. Or42a olfactory receptors required for Or42a OSN critical period remodeling. $\boldsymbol{A}$, Representative confocal images of Or42a -mCD8::GFP in OSNs innervating the ALVM7 glomerulus. Early critical period exposure ( $0-2$ dpe) to the vehicle only (oil, top) or $25 \%$ EB (bottom) in the Or42a-mCD8::GFP genetic background (control, left) and the Or42a-mCD8::GFP; Or42a ${ }^{\text {F04305 }}$ null (Or42a mutant, right). White arrows in EB control indicate axonal membrane retraction punctae. $B$, Quantification of Or42a OSN innervation volume of the VM7 glomerulus for all four conditions. Scatter plots represent all the data points and the mean \pm SEM. Significance from two-way ANOVA with Sidak's multiple-comparisons tests between vehicle and EB conditions within each genotype. Top dashed significance bar represents the interaction between genotype and odor exposure. Not significant: $p>0.05$. ${ }^{* *} p<0.01$. ${ }^{* *} p<0.001$.

olfactory behavioral outputs (Acebes et al., 2011, 2012; Franco et al., 2017; Golovin and Broadie, 2017). We therefore tested whether the specific activation of Or42a receptors is required for critical period synaptic remodeling of Or42a OSNs within the VM7 glomerulus. To test the role of Or42a receptor activity, we compared control animals expressing mCD8::GFP under the Or42a promoter (Or42a-mCD8::GFP) (Stephan et al., 2012) with the characterized Or42a mutants that completely eliminate the receptor response to the $\mathrm{EB}$ odorant (Or42a $a^{F 04305}$, Or42amCD8::GFP) (Olsen et al., 2007). As above, Or42a OSN innervation of the VM7 glomerulus was compared between the odorant vehicle controls (mineral oil) and EB odorant dissolved in the oil at $25 \%$ concentration. The odorant exposure was for $48 \mathrm{~h}$ at $0-2$ dpe, within the initial, early-use critical period. Sample images and quantified VM7 glomerular volume data for the four conditions are shown in Figure 6.

Compared with vehicle controls, EB-treated animals show strong loss of OSN innervation, marked by Or42a-mCD8::GFP punctae characteristic of axonal retraction (Fig. 6A, arrows). Quantification shows a significant effect of EB exposure on Or42a OSN innervation $\left(F_{(1,79)}=\right.$ $13.74, p=0.0004$, two-way ANOVA; Fig. $6 B$, left). Follow up multiple-comparisons tests reveal a significant difference between the vehicle control (oil; $1.0 \pm 0.059$, $n=26)$ and EB-treated animals $(0.543 \pm$ $0.091, n=21)\left(t_{(79)}=5.226, p=4.13 \times\right.$ $10^{-6}$, Sidak's multiple-comparisons test). In sharp contrast, Or $42 a$ mutants exposed to EB exhibit Or42a OSN innervation indistinguishable from oil-exposed mutants (Fig. 6A, right). Membrane punctae characteristic of axonal retraction (Ertürk et al., 2007) are widely present in EB-treated VM7 control glomeruli (Fig. 6A, arrows) but do not occur in EB-treated Or42a mutants (Fig. $6 A$, right), which resembles the vehicle controls in both genotypes. Quantification shows no significant difference between the vehicle control in the Or $42 a$ mutant $(0.817 \pm 0.043, n=19)$ and the EB-treated Or42a mutant (0.783 \pm 0.047 , $n=17)\left(t_{(79)}=0.3427, p=0.980\right.$, Sidak's multiple-comparisons test; Fig. $6 B$, right). The effect of EB exposure on genetic controls versus Or42a mutants is also significant (exposure $\times$ genotype interaction, $F_{(1,79)}=10.19, p=0.002$, two-way ANOVA; Fig. 6B, top dashed bar). These results demonstrate that specific Or42a receptor odorant activation is required for the critical period remodeling of the Or42a OSN innervation of the VM7 glomerulus, suggesting a cell-intrinsic, activity-dependent mechanism.

\section{Activity-dependent modulation of OSN critical period remodeling of glomeruli innervation}

Activity has long been established to mediate critical period neural circuit remodeling, both within specific neuron classes (Devaud et al., 2003b; Sachse et al., 2007) and between different neurons at a circuit level (Acebes et al., 2011; S. Das et al., 2011; Lieber et al., 2011; Sudhakaran et al., 2014). We therefore tested whether activity within the Or42a OSNs specifically, or the activation of downstream neurons via synaptic neurotransmission within the neural circuit, plays a role in critical period remodeling in the VM7 glomerulus. To test both activity levels, we used two widely used transgenic tools: (1) UAS-targeted expression of the tetanus toxin light chain (TeTxLC) protease, which cleaves the vSNARE synaptobrevin to block synaptic vesicle fusion and eliminate neurotransmission (Sweeney et al., 1995); and (2) UAS-targeted expression of the exogenous inward rectifying potassium channel 2.1 (Kir2.1), which increases $\mathrm{K}^{+}$permeability, hyperpolarizes the neuron, and thus inhibits action potential firing within the targeted neuron (Baines et al., 2001). Both UAS constructs were driven with $\mathrm{Or} 42 a-\mathrm{Gal} 4$ only within the targeted Or42A OSNs innervating the VM7 glomerulus. To examine VM7 innervation, Or42a OSNs coexpress the UAS-mCD8::GFP mem- 
brane label. Comparisons were done for each genotype between a vehicle oil control and EB dissolved in the oil at 25\% for 0-2 dpe. Sample images for all conditions and quantified VM7 glomerular innervation for all six conditions are shown in Figure 7.

Transgenic controls respond to EB exposure with a strong decrease in innervation and characteristic axonal punctae (Fig. 7A, left). Quantification again shows the significant effect of odorant exposure $\left(F_{(1,144)}=298.4, p=1.29 \times 10^{-36}\right.$, twoway ANOVA; Fig. 7B, left). Follow-up multiple comparisons reveal a significant decrease of OSN innervation in transgenic controls: oil $1.0 \pm 0.037(n=27)$ versus EB $0.500 \pm 0.062(n=25)\left(t_{(144)}=9.064\right.$, $p=6.22 \times 10^{-15}$, Sidak's multiplecomparisons test). TeTxLC neurotransmission blockade specifically in Or42a OSNs does not prevent critical period remodeling but, rather, results in a further loss of innervation (Fig. 7A, middle). This increased remodeling is accompanied by lack of axon retraction punctae (compare with Fig. 7A, left, arrows), suggesting a more rapid, complete axonal retraction process. In general, axonal punctae are observed less frequently with the greatest innervation loss, suggesting a nearly complete absence of Or42a OSNs within VM7 (Fig. 7A, middle). We hypothesize that TeTxLC has a clear developmental effect on VM7 innervation as the oil TeTxLC condition has a larger Or42a OSN volume compared with controls $\left(t_{(144)}=3.135\right.$, $p=0.014$ Sidak's multiple-comparison test). Quantification with follow up multiple-comparisons test between the oil control TeTxLC condition (1.179 \pm 0.049, $n=22)$ and EB-treated TeTxLC condition $(0.043 \pm 0.011, n=21)$ reveals significant volume reduction $\left(t_{(144)}=\right.$ $18.74, p=1.32 \times 10^{-39}$, Sidak's multiple-comparisons test; Fig. $7 B)$. A linear regression analysis shows that the TeTxLC block significantly increases the effect of EB exposure compared with controls $\left(-0.636 \pm 0.007, t_{(144)}=-7.195, p=2.21 \times 10^{-10}\right.$, two-tailed unpaired $t$ test with Sidak's correction; Fig. $7 B$, middle dashed bar).

We next tested critical period remodeling with the Kir2.1 suppression of action potential firing in the Or42A OSNs. Like the Or42a mutant, EB exposure did not significantly alter OSN innervation in Kir2.1 compared with the vehicle controls (Fig. 7A, right). However, a complication to this interpretation is that Kir2.1 activity suppression in Or42a OSNs strongly reduces VM7 innervation in the vehicle controls (Fig. 7A; compare control and Kir2.1 oil-exposed conditions). The striking effect shows activitydependent control of OSN refinement but may indicate a need for activity for the initial innervation of the VM7 glomerulus (Chiang et al., 2009). Despite the overall effect of Kir2.1 activity suppression on OSN innervation, EB treatment still produces axonal retraction punctae similar to controls (Fig. 7A, Kir2.1 EB).
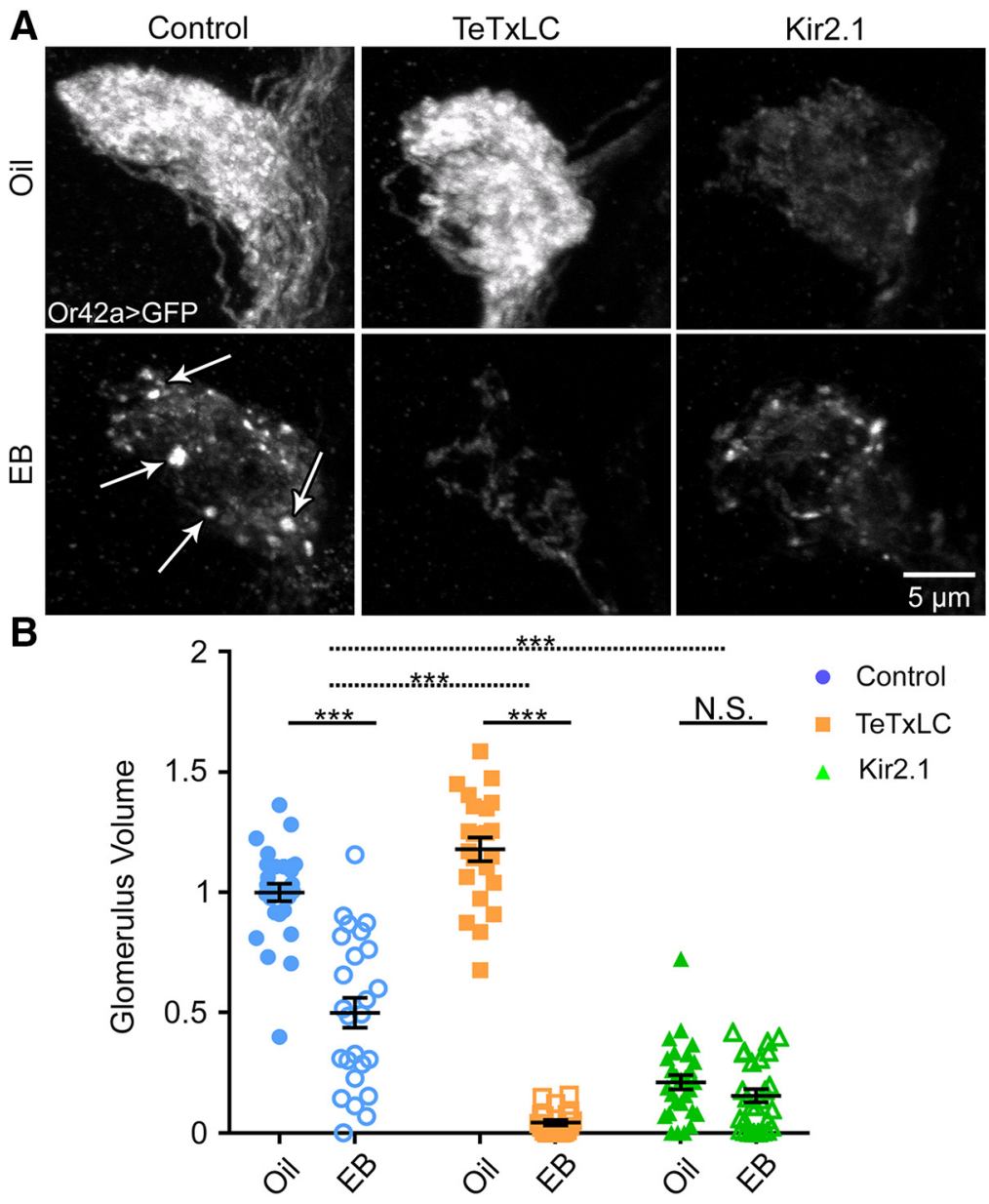

- Control

- TeTXLC

$\Delta$ Kir2.1

Figure 7. Activity-dependent critical period remodeling of olfactory glomeruli innervation. $\boldsymbol{A}$, Representative confocal images of Or42a OSNs innervating the VM7 olfactory glomerulus (Or42a-Gal4-driven UAS-mCD8::GFP) following critical period exposure (0-2 dpe) to vehicle only (oil, top) or 25\% EB (bottom). Three genotypes are compared: (1) the background control $w^{1118}$ (O) Or42a-Gal4/+ (control, left);(2) same genotype with UAS-tetanus toxin light chain (TeTxLC, middle) to block potentials. White arrows in EB control (left) indicate OSN axonal membrane retraction punctae. B , Quantification of Or42a OSN Significance is shown within genotypes from two-way ANOVA with Sidak's multiple-comparisons tests (bottom bars), or from multiple regression with two-tailed unpaired $t$ tests to test interactions between genotype and treatment for TeTxLC (dashed middle bar) and Kir2.1 (dashed top bar) conditions. Not significant $(p>0.05) .{ }^{* * *} p<0.001$.

Quantification of innervation volume shows a strong reduction already in the vehicle control Kir2.1 condition (oil; $0.210 \pm$ $0.030, n=28)$ with no significant loss with EB exposure ( $0.154 \pm$ $0.028, n=27 ; t_{(144)}=1.043, p=0.917$, Sidak's multiplecomparisons test; Fig. $7 B$, right). Linear regression analyses show that Kir2.1 activity suppression attenuates the EB treatment effect compared with controls by $0.441 \pm 0.007$, and is significantly reduced compared with the controls $\left(t_{(144)}=5.327, p=2.63 \times\right.$ $10^{-6}$, two-tailed unpaired $t$ test with Sidak's correction; Fig. $7 B$, top dashed bar). Thus, suppression of Or42A OSN activity reduced baseline innervation and blocks critical period remodeling. Overall, these results indicate that cell-intrinsic OSN activity is important for innervation, and that OSN synaptic signaling limits OSN remodeling during the critical period.

\section{Critical period remodeling requires NMDA glutamatergic transmission, but not GABA signaling}

Given that neurotransmission is important for control of critical period remodeling, we next dissected intercellular signaling mechanisms. Previous work suggests both GABAergic and gluta- 

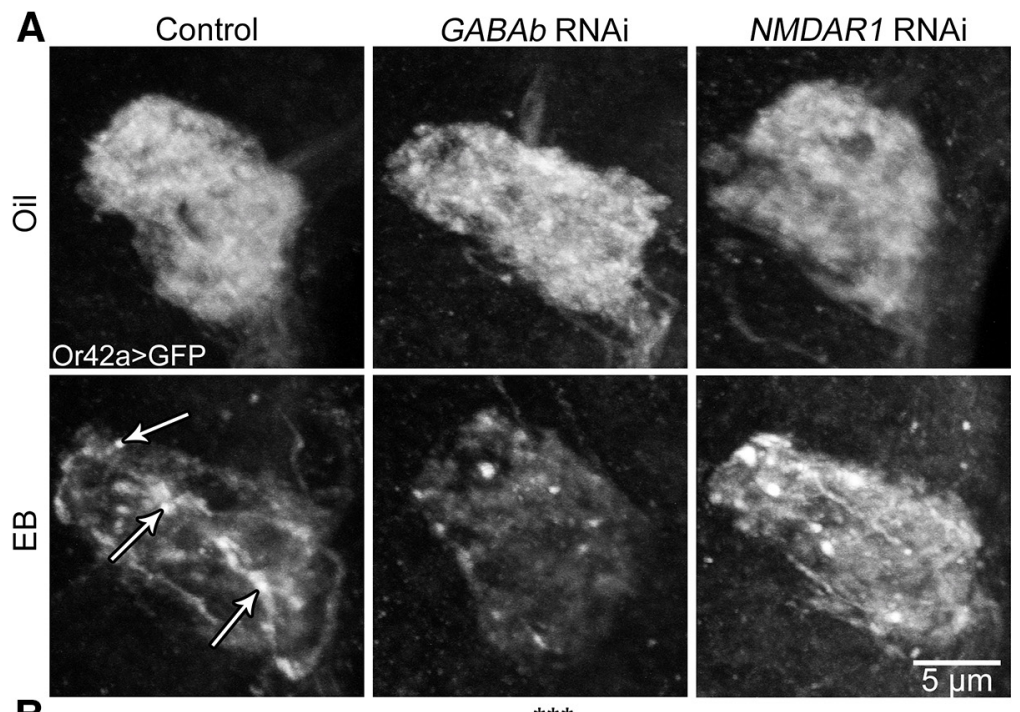

B

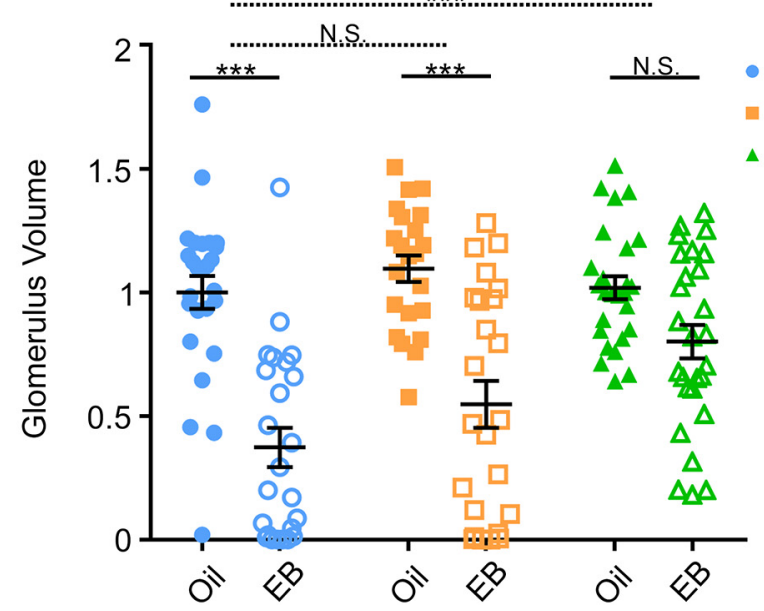

Figure 8. NMDAR glutamatergic transmission required for critical period OSN remodeling. $A$, Representative confocal images of Or42a OSNs innervating the VM7 olfactory glomerulus (Or42a-Gal4-driven UAS-mCD8::GFP) following critical period exposure (0-2 dpe) to vehicle only (oil, top) or 25\% EB (bottom). Three genotypes are compared: (1) the background control $w^{1118}$; UAS-mCD8::GFP/+; Or42a-Gal4/+ (control, left); (2) same genotype with UAS-GABAb R3 RNAi (middle); or (3) UAS-NMDAR1 RNAi (right). White arrows in EB control indicate OSN membrane retraction punctae. $B$, Quantification of Or42a OSN innervation volume of the VM7 glomerulus for all six conditions. Scatter plots represent all the data points and the mean \pm SEM. Significance is shown within genotypes from two-way ANOVA with Sidak's multiple-comparisons tests (bottom bars), or multiple regression with two-tailed unpaired $t$ tests to test the interactions between genotype and odorant treatment for GABAb RNAi (dashed middle bar) and NMDAR1 RNAi (dashed top bar). Not significant $(p>0.05)$. ${ }^{* * *} p<0.001$.

matergic signaling play important roles in regulating $\mathrm{AL}$ dependent behavior (S. Das et al., 2011). Labeling studies indicate that GABAb R2/3 and NMDA R1/R2 receptors are present in the AL (Xia et al., 2005; Raccuglia et al., 2016). To independently test GABAergic and glutamatergic signaling, we used transgenic RNAi to knock down GABA receptors (GABAb R3) and NMDA receptors (NMDAR1) specifically in the Or42a OSNs (Ni et al., 2009). We then assessed Or42a innervation of the VM7 glomerulus as above, comparing oil vehicle controls with 25\% EB during the critical period ( $0-2$ dpe; Fig. 8). A two-way ANOVA shows a significant effect of odorant exposure $\left(F_{(1,143)}=66.27, p=3.5 \times\right.$ $10^{-13}$; Fig. $8 A, B$, left). Compared with controls, GABAb R3 RNAi flies show similar reduction of VM7 innervation after EB exposure (Fig. 8A, middle). Quantification shows that the transgenic control reduction in Or42a OSN volume in VM7 with EB exposure (oil $1.0 \pm 0.067, n=26$, vs EB control $0.373 \pm 0.079$, $n=24 ; t_{(143)}=6.376, p=1.18 \times 10^{-8}$, Sidak's multiplecomparisons test) is similar to the effects in the GABAb R3 RNAi condition (oil $1.096 \pm 0.053, n=22$, vs $\mathrm{EB} 0.547 \pm 0.095, n=24 ; t_{(143)}=5.348$, $p=1.72 \times 10^{-6}$, Sidak's multiplecomparisons test; Fig. $8 B$, middle). There is no significant difference due to $G A B A b$ R3 RNAi on EB reduction compared with controls (average difference $0.0787 \pm$ $0.0126, t_{(143)}=0.5242, p=0.99$, twotailed unpaired $t$ test with Sidak's correction; dashed middle bar).

Given that $G A B A b \quad R 3$-mediated GABAergic signaling has no detectable effect on critical period OSN innervation remodeling, we next assayed roles of NMDAR1-mediated glutamatergic signaling. In the transgenic controls, EB exposure during the critical period ( $0-2$ dpe) results in a highly significant reduction in OSN innervation compared with the odorant vehicle (Fig. $8 A, B$, left). In sharp contrast, NMDAR1 RNAi OSN knockdown strikingly suppresses this EBdependent remodeling (Fig. $8 A$, right). Despite the decreased change in Or42a OSN innervation, the VM7 glomerulus with NMDAR1 RNAi still shows axonal retraction punctae, consistent with an incomplete axonal retraction process in the absence of effective NMDA signaling. Quantification shows that the vehicleexposed NMDAR1 RNAi condition (oil; $1.019 \pm 0.047, n=26$ ) is not significantly different from the EB-treated NMDAR1 RNAi condition $(\mathrm{EB} ; 0.800 \pm 0.068, n=$ $27 ; t_{(143)}=2.284, p=0.114$, Sidak's multiple-comparisons test; Fig. $8 B$, right). NMDAR1 knockdown significantly attenuates the effect of critical period EB exposure on Or42a OSN innervation compared with controls $\left(0.423 \pm 0.0111, t_{(143)}=\right.$ 3.1851, $p=0.0088$, two-tailed unpaired $t$ test with Sidak's correction; Fig. $8 B$, top dashed bar). Glutamatergic interneurons are the only known source of glutamate in the AL (A. Das et al., 2011; Liu and Wilson, 2013), suggesting that these cells are the signal source. These results indicate that NMDA glutamatergic signaling onto OSNs, but not GABAb GABAergic signaling, is important for critical period OSN remodeling.

\section{Wingless/Wnt-1 signaling is not involved in critical period Or42a remodeling}

Other forms of trans-synaptic signaling reportedly play key roles in sensory experience remodeling (Huang et al., 1999; Chiang et al., 2009; Lieber et al., 2011). Wingless (Wg; Wnt-1) is a prominent example, with Wg ligand activating the Drosophila Frizzled 2 $(d F z 2)$ receptor to inhibit Drosophila GSK3 $\beta$ Shaggy (Sgg) and regulate connectivity (Packard et al., 2002; Chiang et al., 2009; Korkut et al., 2009; Tsai et al., 2012; Friedman et al., 2013; Kopke et al., 2017). We used genetic approaches to interrogate this Wg signaling pathway in OSN critical period remodeling. We first removed one copy of $w g\left(w g^{l-17} /+\right)$ (Baker, 1987; Kopke et al., 2017) to reduce the Wg ligand level. In controls, 25\% EB expo- 
A
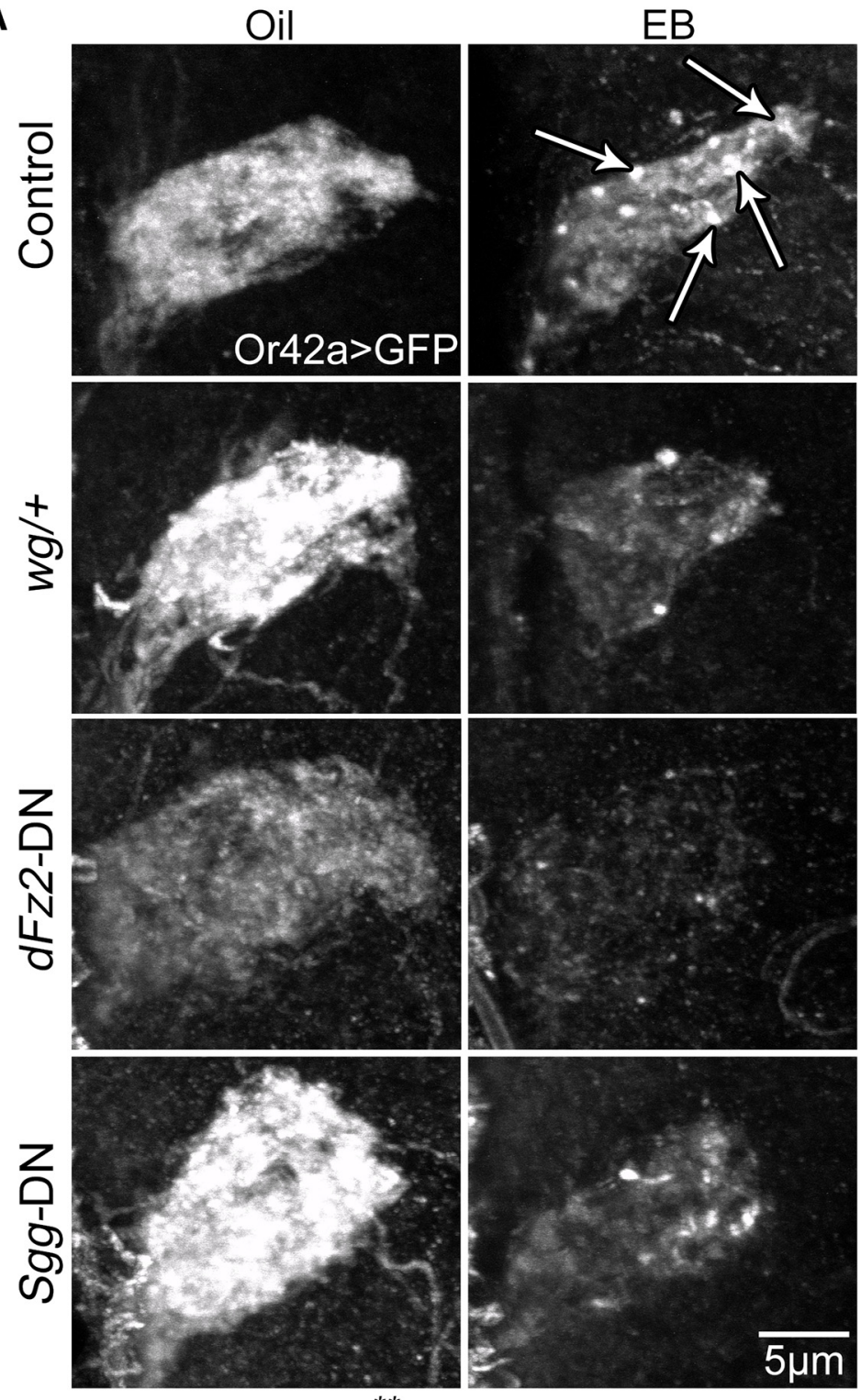

\section{B}

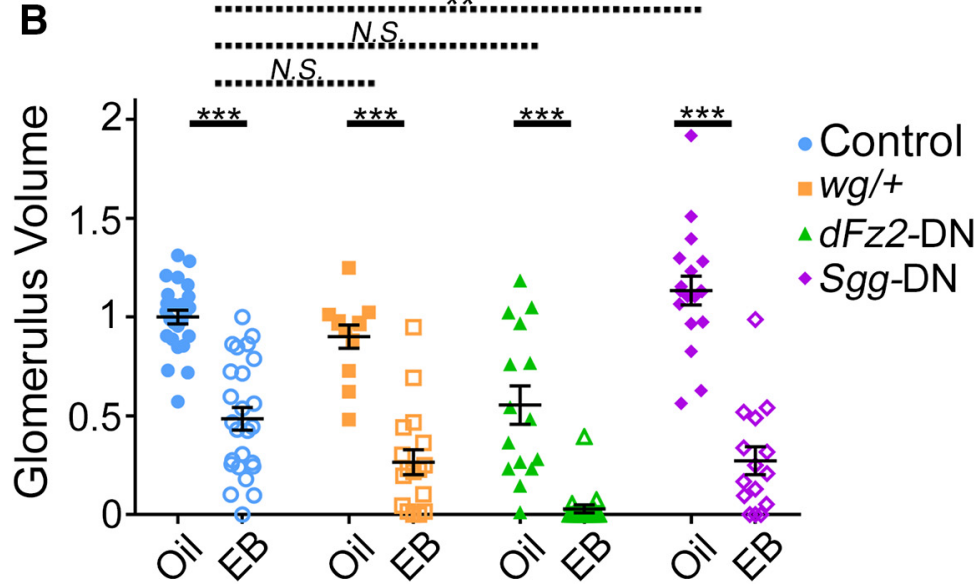

Figure 9. Wnt Wg signaling not involved in critical period-sensitive OSN remodeling. $\boldsymbol{A}$, Representative confocal images of Or42a OSNs innervating the VM7 olfactory glomerulus (Or42a-Gal4-driven UAS-mCD8::GFP) following critical period exposure (0-2 dpe) to vehicle only (oil, left) or $25 \%$ EB (right). Four genotypes are compared: (1) background control $w^{1118} ;$ UAS-mCD8: $\mathrm{GFP} /+; 0$ r42a-Gal4/+ (control, top); (2) same genotype with a heterozygous wingless null $\left(w g^{l-17} /+\right.$, second row); (3) same genotype with UAS-Drosophila frizzled-2 dominant negative (dFz2-DN, third row); or (4) the UAS-shaggy dominant negative (SggDN, bottom). White arrows in EB control indicate OSN axonal membrane retraction punctae. Female brains were used for these experiments. $\boldsymbol{B}$, Quantification of OSN innervation volume of the VM7 glomerulus for all eight conditions. Scatter plots represent al sure in the critical period ( $0-2$ dpe) causes the expected loss of OSN innervation and appearance of axonal retraction punctae (Fig. 9A, top, arrows), and quantification shows the typical loss of VM7 glomerulus innervation: oil $1.0 \pm 0.035(n=25), \mathrm{EB}$ $0.485 \pm 0.058(n=25)\left(t_{(138)}=7.093\right.$, $p=4.37 \times 10^{-10}$, Sidak's multiplecomparisons test; odorant exposure, effects $F_{(1,138)}=210.8, p=2.84 \times 10^{-29}$, two-way ANOVA; Fig. 9B, left). Heterozygous $w g^{l-17} /+$ does not impact the effect of EB exposure on OSNs, with innervation indistinguishable from matched controls (Fig. 9A). Quantification shows a significant difference between vehicle and EB exposure: oil $w g^{l-17} /+0.901 \pm 0.059$ $(n=12)$ versus EB-treated $w g^{l-17} /+0.266 \pm$ $0.063(n=17)\left(t_{(138)}=6.564, \mathrm{p} 6.86 \times\right.$ $10^{-9}$, Sidak's multiple-comparisons test; Fig. 9B). There is no significant effect on EB exposure between $w g^{1-17} /+$ and controls $\left(0.120 \pm 0.009, t_{(138)}=-1.0997, p=0.893\right.$, two-tailed unpaired $t$ test on interaction terms from linear regression with Sidak's correction; Fig. 9B, third dashed bar).

Despite the lack of Wg effect, we used stronger transgenic manipulations to further test the signaling pathway. We targeted a $d F z 2$ dominant-negative ( $d F z 2$ DN) (Zhang and Carthew, 1998) to Or42a OSNs. This reduces VM7 innervation in vehicle controls (Fig. 9A) but does not significantly impact the effect of EB exposure: oil $0.554 \pm 0.097(n=15)$ versus $\mathrm{EB}$ $0.029 \pm 0.021(n=19)\left(t_{(138)}=5.926\right.$, $p=1.65 \times 10^{-7}$, Sidak's multiplecomparisons test; Fig. 9B). In $d F z 2-D N$ flies, the EB treatment effect compared with controls is weakly augmented by $-0.0104 \pm 0.01023$, which is not significantly different $\left(t_{(138)}=-0.086, p=\right.$ 0.999 , two-tailed unpaired $t$ test with $\mathrm{Si}$ dak's correction; Fig. 9B, second dashed bar). We also expressed an $S g g$ dominantnegative (Sgg-DN) (Bourouis, 2002) in Or42a OSNs to inhibit Wg signaling. Compared with controls, Sgg-DN increases the effect of critical period EB exposure on innervation (Fig. $9 A$, bottom). Quantification shows that vehicle controls $(1.135 \pm 0.073, n=18)$ are significantly different from EB-treated $S g g$-DN $\left(0.273 \pm 0.070, n=15 ; t_{(138)}=9.607, p=\right.$

$\leftarrow$

the data points and the mean \pm SEM. Significance within genotypes from two-way ANOVA with Sidak's multiplecomparisons tests (bottom bars), or from multiple regression with two-tailed unpaired $t$ tests to test interactions between genotype and treatment for Sgg-DN (dashed top bar), dFz2-DN (dashed second bar), and $w g /+$ (dashed third bar). Not significant $(p>0.05)$. ${ }^{* *} p<0.01 .{ }^{* *} p<0.001$. 
$3.48 \times 10^{-16}$, Sidak's multiple-comparisons test; Fig. 9B, right). In $S g g$-DN flies, the OSN volume reduction from EB exposure compared with control is augmented by $-0.347 \pm 0.0104$, which is significant $\left(t_{(138)}=-2.849, p=0.035\right.$, two-tailed unpaired $t$ test with Sidak's correction; Fig. $9 B$, first dashed bar). However, an Sgg role in remodeling is likely independent of $\mathrm{Wg}$ signaling, as the other Wg manipulations do not impact remodeling, other pathways regulate Sgg activity, and Sgg independently affects glomerulus synapse number and behavior (Acebes et al., 2011). Overall, Wg signaling does not appear to be involved in regulating EB-dependent critical period remodeling.

\section{Discussion}

In Drosophila, the first few days of sensory experience are a usedependent critical period for central olfactory circuit refinement with a greatly heightened capacity for synaptic remodeling (Devaud et al., 2003a; Sachse et al., 2007; Tessier and Broadie, 2009; Doll and Broadie, 2015, 2016; Doll et al., 2017). Here, we test this early posteclosion time window $(0-2 \mathrm{~d})$ in contrast to maturity (7-9 d). We find that odorant exposure only during the early period causes a striking reduction of Or42a OSN innervation onto their target VM7 synaptic glomerulus. This remodeling is odorant concentration-dependent and developmentally regulated. Despite early work clearly showing that critical period odorant experience reduces glomerulus innervation volume (Devaud et al., 2001, 2003a,b), no other work, to our knowledge, has shown an odorant-dependent decrease in OSN glomerular innervation, and only previous work from our laboratory has demonstrated reduction at the level of the postsynaptic projection neurons (Doll and Broadie, 2015). The current study thus refutes the conclusion that glomeruli activated by an odorant during the critical period always respond with an innervation increase and presents a more complicated landscape. The current study also reveals larger-magnitude innervation remodeling changes in comparison with earlier studies reporting reductions approaching $\sim 30 \%$ (Devaud et al., 2001, 2003a). These differences likely reflect dosage-dependent effects (10\% vs $15 \%$ or $25 \%$ concentration), and the more careful monitoring of critical period timing.

Similar to a previous report on $\mathrm{CO}_{2}$-sensitive olfactory sensory neurons, we demonstrate that Or42a innervation remodeling is reversible with removal from the odorant (Sachse et al., 2007). Removing animals from EB for 2 or 3 d during the critical period leads to complete reversal of lost glomerulus innervation. Importantly, however, a few animals with $2 \mathrm{~d}$ reversal do not manifest reinnervation. This result suggests that remodeling capacity is reduced with odorant exposure time and the critical period endpoint threshold prevents reversal remodeling to make the OSN glomerulus innervation largely permanent. This conclusion agrees with classical critical period studies (Hubel and Wiesel, 1970; Blakemore et al., 1978; Ma et al., 2014; Tsai and Barnea, 2014; Huang et al., 2015), and is supported by the all-ornothing nature of the reversal. We test synaptic remodeling using OSN-targeted STaR synapse labeling (Chen et al., 2014). Using the STaR technique, we show that odorant-induced synapse elimination is more extensive than process loss, suggesting that loss of synaptic connections precedes axon retraction. miniSOG electron microscopy confirms synapse elimination (Devaud et al., 2001). This loss of synaptic connectivity likely modifies odorant-induced behaviors following EB exposure during the critical period (S. Das et al., 2011; Sudhakaran et al., 2014), which results in long-term habituation with flies avoiding the EB odorant more weakly than naive flies.
We used three manipulations to test the activity dependence of critical period remodeling. First, using Or $42 a^{F 04305}$ receptor mutants that render OSNs EB-insensitive (Olsen et al., 2007), we find that critical period refinement is eliminated. This result indicates that Or42a receptor activity is necessary in a cell-intrinsic mechanism controlling activity specifically within the Or42a OSNs. Second, expressing the tetanus protease to block synaptic vesicle fusion and neurotransmission (Sweeney et al., 1995), we find that OSN synaptic output is not only dispensable for the odorant-induced remodeling but also acts to limit the extent of glomerular retraction. This intriguing finding suggests a mechanism acting trans-synaptically to limit critical period refinement of Or42a OSN innervation of VM7 glomerulus. A strong candidate is Notch signaling, which was recently shown to mediate Or82a OSN remodeling (Kidd and Lieber, 2016). Third, targeted Kir2.1 expression to suppress cell-intrinsic OSN electrical activity (Baines et al., 2001) blocks critical period innervation remodeling, similarly to the loss of Or42a receptor function. However, Kir2.1 silencing reduces glomerular innervation in both the vehicle- and EB-exposed animals, suggesting that activity is required for OSN axonal maintenance (Chiang et al., 2009). Together, these results demonstrate that activity-dependent critical period remodeling requires OSN cell-autonomous olfactory receptor activity modulated by downstream neuronal communication within the neural circuit.

The loss of critical period remodeling in OSNs lacking the Or42a EB odorant receptor indicates that the mechanism requires olfactory reception in the specific OSN, and lateral communication between different OSN classes within the AL is not sufficient to cause remodeling (Acebes et al., 2011, 2012; Franco et al., 2017; Golovin and Broadie, 2017). The maintenance of OSN critical period remodeling with tetanus toxin blockade does not exclude electrical synapse involvement, which ise not affected by the neurotoxin (Sweeney et al., 1995). However, OSN electrical synapses have not been described, and electrical synapse loss does not alter OSN function (Yaksi and Wilson, 2010). Nevertheless, innexin mutants could be used to test possible electrical synapse roles (Phelan et al., 1996). Our studies indicate that tetanus toxin has a consequence on OSN development because animals have increased glomerulus innervation in the vehicle controls. This confirms previous results that tetanus toxin produces increased axon branching in olfactory projection neurons (Doll et al., 2017). Interpretation of our Kir2.1 result is likewise complicated by decreased Or42a OSN-VM7 innervation in vehicle controls. Although remodeling is eliminated with Kir2.1 silencing, the assay may not be sensitive enough to detect changes with the reduced innervation. Future studies using optogenetics to conditionally silence OSNs in the critical period may avoid the developmental effects of constitutive Kir2.1 silencing.

Our results indicate that NMDAR1-mediated glutamatergic neurotransmission is required for critical period OSN remodeling, consistent with previous work showing that NMDAR1 is required for glomerulus volume increases and long-term habituation after EB exposure (S. Das et al., 2011). The only known source of AL glutamate release is a population of ventral local interneurons (A. Das et al., 2011), suggesting that these interneurons regulate critical period remodeling. GABAergic signaling also regulates Or42a OSN activity (Olsen and Wilson, 2008; Raccuglia et al., 2016), but our results indicate that AL-expressed GABAb R3 receptors (Okada et al., 2009) are not necessary for remodeling. This finding is consistent with previous reports that GABAergic signaling impacts long-term habituation, but not AL 
circuit changes (S. Das et al., 2011). We conclude that NMDA receptor, but not GABAb receptor, signaling combines with odorant-dependent activity in Or42a OSNs to drive synaptic remodeling during the critical period. The role of activity and NMDA receptor signaling is in agreement with previous studies on OSN response to odorant exposure (S. Das et al., 2011). However, the direction and magnitude of remodeling changes reported here differ from earlier reports. The loss of Or42a OSN VM7 innervation is somewhat reminiscent of the progressive loss of axonal processes in Or83b (orco) mutants that lack olfactory sensory reception (Chiang et al., 2009). These orco mutants also display axonal punctae similar to the mCD8::GFP membrane retractions reported here.

The punctae in retracting OSN axon processes closely resemble mammalian retraction bulbs (Li and Raisman, 1995). However, for this characterization, we would need to establish that the punctae are long-lived and display a disorganized microtubule architecture (Ertürk et al., 2007). Despite the striking similarities between EB-exposed OSNs and orco mutants, the time course is enormously different, with odorant-dependent critical period remodeling occurring immediately after eclosion $(0-2$ dpe) and orco retraction phenotypes starting much later (Chiang et al., 2009). Also, unlike the orco mutant retraction, critical period refinement does not involve $\mathrm{Wg}$ signaling (Packard et al., 2002; Chiang et al., 2009; Korkut et al., 2009; Tsai et al., 2012), as both $W g$ heterozygotes and targeted $d F z 2-\mathrm{DN}$ in Or42a OSNs fail to impact axon retraction. Curiously, targeted $S g g$-DN in Or42a OSNs enhances odorantinduced critical period remodeling, with a greater degree of VM7 innervation loss following EB exposure. However, $S g g$-DN effects likely do not act via the Wg signaling pathway, as this mechanism would be expected to limit OSN innervation reduction. Importantly, Sgg acts independently to regulate glomerulus synapse number (Acebes et al., 2011). The Wg pathway studies reported here support a role in the maintenance of OSN glomerular innervation but do not indicate a role in the regulation of critical period remodeling.

This study demonstrates a novel mode of odorant-dependent critical period remodeling of brain olfactory circuitry. EB odorant exposure during an early-use critical period causes an extensive retraction of Or42a OSN innervation of the AL VM7 glomerulus. This striking loss of innervation is independent of changes in number of Or42a OSNs, consistent with previous reports that show glomerular innervation changes occur independently of the peripheral OSN cell bodies (Sachse et al., 2007; Chiang et al., 2009). The reduction of axonal innervation is presaged by striking elimination of OSN synaptic connections. Ultrastructural studies confirm synapse elimination due to heightened critical period odorant experience. Synapse remodeling requires Or42a receptor activity and NMDA receptor signaling. These results extend our knowledge of AL critical period remodeling by demonstrating how activity diminishes innervation of an olfactory glomerulus. At a molecular level, this remodeling likely relies on an unidentified trans-synaptic signal. The previously identified Wingless signaling pathway is not detectably involved (Chiang et al., 2009), and Notch trans-synaptic signaling is the future candidate (Kidd and Lieber, 2016). This work has important implications for our understanding of critical period remodeling of sensory circuits and provides a step toward our understanding of the developing nervous system in both health and disease.

\section{References}

Acebes A, Martín-Peña A, Chevalier V, Ferrús A (2011) Synapse loss in olfactory local interneurons modifies perception. J Neurosci 31:2734-2745.

Acebes A, Devaud JM, Arnés M, Ferrús A (2012) Central adaptation to odorants depends on PI3K levels in local interneurons of the antennal lobe. J Neurosci 32:417-422.

Baines RA, Uhler JP, Thompson A, Sweeney ST, Bate M (2001) Altered electrical properties in Drosophila neurons developing without synaptic transmission. J Neurosci 21:1523-1531.

Baker NE (1987) Molecular cloning of sequences from wingless, a segment polarity gene in Drosophila: the spatial distribution of a transcript in embryos. EMBO J 6:1765-1773.

Baroncelli L, Scali M, Sansevero G, Olimpico F, Manno I, Costa M, Sale A (2016) Experience affects critical period plasticity in the visual cortex through an epigenetic regulation of histone post-translational modifications. J Neurosci 36:3430-3440.

Blakemore C, Garey LJ, Vital-Durand F (1978) The physiological effects of monocular deprivation and their reversal in the monkey's visual cortex. J Physiol 283:223-262.

Bourouis M (2002) Targeted increase in shaggy activity levels blocks wingless signaling. Genesis 34:99-102.

Chen Y, Akin O, Nern A, Tsui CY, Pecot MY, Zipursky SL (2014) Cell-typespecific labeling of synapses in vivo through synaptic tagging with recombination. Neuron 81:280-293.

Chiang A, Priya R, Ramaswami M, Vijayraghavan K, Rodrigues V (2009) Neuronal activity and wnt signaling act through Gsk3- $\beta$ to regulate axonal integrity in mature Drosophila olfactory sensory neurons. Development 136:1273-1282.

Contractor A, Klyachko VA, Portera-Cailliau C (2015) Altered neuronal and circuit excitability in fragile X syndrome. Neuron 87:699-715.

Couto A, Alenius M, Dickson BJ (2005) Molecular, anatomical and functional organization of the Drosophila olfactory system. Curr Biol 15:15351547.

Das A, Chiang A, Davla S, Priya R, Reichert H, VijayRaghavan K, Rodrigues V (2011) Identification and analysis of a glutamatergic local interneuron lineage in the adult Drosophila olfactory system. Neural Syst Circuits 1:4.

Das S, Sadanandappa MK, Dervan A, Larkin A, Lee J, Sudhakaran IP, Priya R, Heidari R, Holohan EE, Pimentel A, Gandhi A, Ito K, Sanyal S, Wang JW, Rodrigues V, Ramaswami M (2011) Plasticity of local GABAergic interneurons drives olfactory habituation. Proc Natl Acad Sci U S A 108: $646-654$.

Devaud JM, Acebes A, Ferrús A (2001) Odor exposure causes central adaptation and morphological changes in selected olfactory glomeruli in Drosophila. J Neurosci 21:6274-6282.

Devaud JM, Acebes A, Ramaswami M, Ferrús A (2003a) Structural and functional changes in the olfactory pathway of adult Drosophila take place at a critical age. J Neurobiol 56:13-23.

Devaud JM, Keane J, Ferrús A (2003b) Blocking sensory inputs to identified antennal glomeruli selectively modifies odorant perception in Drosophila. J Neurobiol 56:1-12.

Dölen G, Osterweil E, Rao BS, Smith GB, Auerbach BD, Chattarji S, Bear MF (2007) Correction of fragile X syndrome in mice. Neuron 56:955-962.

Doll CA, Broadie K (2015) Activity-dependent FMRP requirements in development of the neural circuitry of learning and memory. Development 142:1346-1356

Doll CA, Broadie K (2016) Neuron class-specific requirements for fragile X mental retardation protein in critical period development of calcium signaling in learning and memory circuitry. Neurobiol Dis 89:76-87.

Doll CA, Vita DJ, Broadie K (2017) Fragile X mental retardation protein requirements in activity-dependent critical period neural circuit refinement. Curr Biol 27:2318-2330.e3.

Ertürk A, Hellal F, Enes J, Bradke F (2007) Disorganized microtubules underlie the formation of retraction bulbs and the failure of axonal regeneration. J Neurosci 27:9169-9180.

Fielde AM (1904) Power of recognition among ants. Biol Bull 7:227-250.

Fishilevich E, Vosshall LB (2005) Genetic and functional subdivision of the Drosophila antennal lobe. Curr Biol 15:1548-1553.

Flockhart I, Booker M, Kiger A, Boutros M, Armknecht S, Ramadan N, Richardson K, Xu A, Perrimon N, Mathey-Prevot B (2006) FlyRNAi: the Drosophila RNAi screening center database. Nucleic Acids Res 34:D489D494. 
Franco LM, Okray Z, Linneweber GA, Hassan BA, Yaksi E (2017) Reduced lateral inhibition impairs olfactory computations and behaviors in a Drosophila model of fragile X syndrome. Curr Biol 27:1111-1123.

Friedman SH, Dani N, Rushton E, Broadie K (2013) Fragile X mental retardation protein regulates trans-synaptic signaling in Drosophila. Dis Model Mech 6:1400-1413.

Golovin RM, Broadie K (2016) Developmental experience-dependent plasticity in the first synapse of the Drosophila olfactory circuit. J Neurophysiol 116:2730-2738.

Golovin RM, Broadie K (2017) Neural circuits: reduced inhibition in fragile X syndrome. Curr Biol 27:298-300.

Grabe V, Baschwitz A, Dweck HK, Lavista-Llanos S, Hansson BS, Sachse S (2016) Elucidating the neuronal architecture of olfactory glomeruli in the Drosophila antennal lobe. Cell Rep 16:3401-3413.

Hallem EA, Carlson JR (2006) Coding of odors by a receptor repertoire. Cell $125: 143-160$

Hara C, Morishita K, Takayanagi-Kiya S, Mikami A, Uchino K, Sakurai T, Kanzaki R, Sezutsu H, Iwami M, Kiya T (2017) Refinement of ectopic protein expression through the GAL4/UAS system in bombyx mori: application to behavioral and developmental studies. Sci Rep 7:11795.

Hensch TK (2005) Critical period plasticity in local cortical circuits. Nat Rev Neurosci 6:877-888.

Hensch TK, Bilimoria PM (2012) Re-opening windows: manipulating critical periods for brain development. Cerebrum 2012:11.

Huang X, Stodieck SK, Goetze B, Cui L, Wong MH, Wenzel C, Hosang L, Dong Y, Löwel S, Schlüter OM (2015) Progressive maturation of silent synapses governs the duration of a critical period. Proc Natl Acad Sci U S A 112:E3131-E3140.

Huang ZJ, Kirkwood A, Pizzorusso T, Porciatti V, Morales B, Bear MF, Maffei L, Tonegawa S (1999) BDNF regulates the maturation of inhibition and the critical period of plasticity in mouse visual cortex. Cell 98:739-755.

Hubel DH, Wiesel TN (1970) The period of susceptibility to the physiological effects of unilateral eye closure in kittens. J Physiol 206:419-436.

Jefferis GS, Marin EC, Stocker RF, Luo L (2001) Target neuron prespecification in the olfactory map of Drosophila. Nature 414:204-208.

Jefferis GS, Marin EC, Watts RJ, Luo L (2002) Development of neuronal connectivity in Drosophila antennal lobes and mushroom bodies. Curr Opin Neurobiol 12:80-86.

Jefferis GS, Vyas RM, Berdnik D, Ramaekers A, Stocker RF, Tanaka NK, Ito K, Luo L (2004) Developmental origin of wiring specificity in the olfactory system of Drosophila. Development 131:117-130.

Jin X, Pokala N, Bargmann CI (2016) Distinct circuits for the formation and retrieval of an imprinted olfactory memory. Cell 164:632-643.

Kidd S, Lieber T (2016) Mechanism of notch pathway activation and its role in the regulation of olfactory plasticity in Drosophila melanogaster. PLoS One 11:e0151279.

Kidd S, Struhl G, Lieber T (2015) Notch is required in adult Drosophila sensory neurons for morphological and functional plasticity of the olfactory circuit. PLoS Genet 11:e1005244.

Kopke DL, Lima SC, Alexandre C, Broadie K (2017) Notum coordinates synapse development via extracellular regulation of wnt wingless transsynaptic signaling. Development 144:3499-3510.

Korkut C, Ataman B, Ramachandran P, Ashley J, Barria R, Gherbesi N, Budnik V (2009) Trans-synaptic transmission of vesicular wnt signals through Evi/Wntless. Cell 139:393-404.

LaLonde M, Janssens H, Yun S, Crosby J, Redina O, Olive V, Altshuller YM, Choi SY, Du G, Gergen JP, Frohman MA (2006) A role for phospholipase D in Drosophila embryonic cellularization. BMC Dev Biol 6:60.

Larsson MC, Domingos AI, Jones WD, Chiappe ME, Amrein H, Vosshall LB (2004) Or83b encodes a broadly expressed odorant receptor essential for Drosophila olfaction. Neuron 43:703-714.

LeVay S, Wiesel TN, Hubel DH (1980) The development of ocular dominance columns in normal and visually deprived monkeys. J Comp Neurol 191:1-51.

Li Y, Raisman G (1995) Sprouts from cut corticospinal axons persist in the presence of astrocytic scarring in long-term lesions of the adult rat spinal cord. Exp Neurol 134:102-111.

Lieber T, Kidd S, Struhl G (2011) DSL-notch signaling in the Drosophila brain in response to olfactory stimulation. Neuron 69:468-481.

Ling D, Salvaterra PM (2011) Robust RT-qPCR data normalization: validation and selection of internal reference genes during post-experimental data analysis. PLoS One 6:e17762.
Liu WW, Wilson RI (2013) Glutamate is an inhibitory neurotransmitter in the Drosophila olfactory system. Proc Natl Acad Sci USA 110:10294-10299.

Livak KJ, Schmittgen TD (2001) Analysis of relative gene expression data using real-time quantitative PCR and the $2-\Delta \Delta C$ T method. Methods 25:402-408.

Ma L, Wu Y, Qiu Q, Scheerer H, Moran A, Yu CR (2014) A developmental switch of axon targeting in the continuously regenerating mouse olfactory system. Science 344:194-197.

Maya Vetencourt J, Sale A, Viegi A, Baroncelli L, De Pasquale R, O’Leary OF, Castrén E, Maffei L (2008) The antidepressant fluoxetine restores plasticity in the adult visual cortex. Science 320:385-388.

McGee AW, Yang Y, Fischer QS, Daw NW, Strittmatter SM (2005) Experience-driven plasticity of visual cortex limited by myelin and Nogo receptor. Science 309:2222-2226.

Morishita H, Miwa JM, Heintz N, Hensch TK (2010) Lynx1, a cholinergic brake, limits plasticity in adult visual cortex. Science 330:1238-1240.

Mosca TJ, Luo L (2014) Synaptic organization of the Drosophila antennal lobe and its regulation by the teneurins. Elife 3:e03726.

Münch D, Galizia CG (2016) DoOR 2.0 comprehensive mapping of Drosophila melanogaster odorant responses. Sci Rep 6:21841.

Ng J, Browning A, Lechner L, Terada M, Howard G, Jefferis GS (2016) Genetically targeted 3D visualisation of Drosophila neurons under electron microscopy and x-ray microscopy using miniSOG. Sci Rep 6:srep38863.

Ni JQ, Liu LP, Binari R, Hardy R, Shim HS, Cavallaro A, Booker M, Pfeiffer BD, Markstein M, Wang H, Villalta C, Laverty TR, Perkins LA, Perrimon N (2009) A Drosophila resource of transgenic RNAi lines for neurogenetics. Genetics 182:1089-1100.

Okada R, Awasaki T, Ito K (2009) Gamma aminobutyric acid (GABA) mediated neural connections in the Drosophila antennal lobe. J Comp Neurol 514:74-91.

Olsen SR, Wilson RI (2008) Lateral presynaptic inhibition mediates gain control in an olfactory circuit. Nature 452:956-960.

Olsen SR, Bhandawat V, Wilson RI (2007) Excitatory interactions between olfactory processing channels in the Drosophila antennal lobe. Neuron 54:89-103.

Packard M, Koo ES, Gorczyca M, Sharpe J, Cumberledge S, Budnik V (2002) The Drosophila wnt, wingless, provides an essential signal for pre- and postsynaptic differentiation. Cell 111:319-330.

Phelan P, Nakagawa M, Wilkin MB, Moffat KG, O'Kane CJ, Davies JA, Bacon JP (1996) Mutations in shaking-B prevent electrical synapse formation in the Drosophila giant fiber system. J Neurosci 16:1101-1113.

Raccuglia D, McCurdy LY, Demir M, Gorur-Shandilya S, Kunst M, Emonet T, Nitabach MN (2016) Presynaptic GABA receptors mediate temporal contrast enhancement in Drosophila olfactory sensory neurons and modulate odor-driven behavioral kinetics. eNeuro 3:ENEURO.0080-16.2016.

Remy JJ, Hobert O (2005) An interneuronal chemoreceptor required for olfactory imprinting in C. elegans. Science 309:787-790.

Sachse S, Rueckert E, Keller A, Okada R, Tanaka NK, Ito K, Vosshall LB (2007) Activity-dependent plasticity in an olfactory circuit. Neuron 56 : $838-850$.

Schindelin J, Arganda-Carreras I, Frise E, Kaynig V, Longair M, Pietzsch T, Preibisch S, Rueden C, Saalfeld S, Schmid B, Tinevez JY, White DJ, Hartenstein V, Eliceiri K, Tomancak P, Cardona A (2012) Fiji: an opensource platform for biological-image analysis. Nat Methods 9:676-682.

Schneider CA, Rasband WS, Eliceiri KW (2012) NIH image to ImageJ: 25 years of image analysis. Nat Methods 9:671-675.

Sears JC, Broadie K (2018) Fragile X mental retardation protein regulates activity-dependent membrane trafficking and trans-synaptic signaling mediating synaptic remodeling. Front Mol Neurosci 10:440.

Semmelhack JL, Wang JW (2009) Select Drosophila glomeruli mediate innate olfactory attraction and aversion. Nature 459:218-223.

Shu X, Lev-Ram V, Deerinck TJ, Qi Y, Ramko EB, Davidson MW, Jin Y, Ellisman MH, Tsien RY (2011) A genetically encoded tag for correlated light and electron microscopy of intact cells, tissues, and organisms. PLoS Biol 9:e1001041.

Stephan D, Sánchez-Soriano N, Loschek LF, Gerhards R, Gutmann S, Storchova Z, Prokop A, Kadow IC (2012) Drosophila Psidin regulates olfactory neuron number and axon targeting through two distinct molecular mechanisms. J Neurosci 32:16080-16094.

Strickfaden H, Xu Z, Hendzel MJ (2015) Visualization of miniSOG tagged 
DNA repair proteins in combination with electron spectroscopic imaging (ESI). J Vis Exp 24:103.

Sudhakaran IP, Hillebrand J, Dervan A, Das S, Holohan EE, Hülsmeier J, Sarov M, Parker R, VijayRaghavan K, Ramaswami M (2014) FMRP and ataxin-2 function together in long-term olfactory habituation and neuronal translational control. Proc Natl Acad Sci U S A 111:99-108.

Sweeney ST, Broadie K, Keane J, Niemann H, O’Kane CJ (1995) Targeted expression of tetanus toxin light chain in Drosophila specifically eliminates synaptic transmission and causes behavioral defects. Neuron 14:341-351.

Tessier CR, Broadie K (2008) Drosophila fragile X mental retardation protein developmentally regulates activity-dependent axon pruning. Development 135:1547-1557.

Tessier CR, Broadie K (2009) Activity-dependent modulation of neural circuit synaptic connectivity. Front Mol Neurosci 2:8.

Tessier CR, Broadie K (2011) The fragile X mental retardation protein developmentally regulates the strength and fidelity of calcium signaling in Drosophila mushroom body neurons. Neurobiol Dis 41:147-159.

Thibault ST, Singer MA, Miyazaki WY, Milash B, Dompe NA, Singh CM, Buchholz R, Demsky M, Fawcett R, Francis-Lang HL, Ryner L, Cheung LM, Chong A, Erickson C, Fisher WW, Greer K, Hartouni SR, Howie E, Jakkula L, Joo D, et al. (2004) A complementary transposon tool kit for Drosophila melanogaster using P and piggyBac. Nat Genet 36:283-287.

Tsai L, Barnea G (2014) A critical period defined by axon-targeting mechanisms in the murine olfactory bulb. Science 344:197-200.

Tsai PI, Wang M, Kao HH, Cheng YJ, Lin YJ, Chen RH, Chien CT (2012) Activity-dependent retrograde laminin A signaling regulates synapse growth at Drosophila neuromuscular junctions. Proc Natl Acad Sci U S A 109:17699-17704.
Vita DJ, Broadie K (2017) ESCRT-III membrane trafficking misregulation contributes to fragile X syndrome synaptic defects. Sci Rep 7:8683.

Vosshall LB, Wong AM, Axel R (2000) An olfactory sensory map in the fly brain. Cell 102:147-159.

Wagh DA, Rasse TM, Asan E, Hofbauer A, Schwenkert I, Dürrbeck H, Buchner S, Dabauvalle MC, Schmidt M, Qin G, Wichmann C, Kittel R, Sigrist SJ, Buchner E (2006) Bruchpilot, a protein with homology to ELKS/ CAST, is required for structural integrity and function of synaptic active zones in Drosophila. Neuron 49:833-844.

Wang JW, Wong AM, Flores J, Vosshall LB, Axel R (2003) Two-photon calcium imaging reveals an odor-evoked map of activity in the fly brain. Cell 112:271-282

Wang JW, Beck ES, McCabe BD (2012) A modular toolset for recombination transgenesis and neurogenetic analysis of Drosophila. PLoS One 7:e42102.

Wilson RI (2013) Early olfactory processing in Drosophila: mechanisms and principles. Annu Rev Neurosci 36:217-241.

Winkle CC, Gupton SL (2016) Membrane trafficking in neuronal development: ins and outs of neural connectivity. Int Rev Cell Mol Biol 322:247-280.

Xia S, Miyashita T, Fu TF, Lin WY, Wu CL, Pyzocha L, Lin IR, Saitoe M, Tully T, Chiang AS (2005) NMDA receptors mediate olfactory learning and memory in Drosophila. Curr Biol 15:603-615.

Yaksi E, Wilson RI (2010) Electrical coupling between olfactory glomeruli. Neuron 67:1034-1047.

Zhang J, Carthew RW (1998) Interactions between wingless and DFz2 during Drosophila wing development. Development 125:3075-3085. 